\title{
A Receptor-Like Inositol Lipid Phosphatase Is Required for the Maturation of Developing Cochlear Hair Bundles
}

\author{
R. J. Goodyear, ${ }^{1}$ P. K. Legan, ${ }^{1}$ M. B. Wright, ${ }^{2}$ W. Marcotti, ${ }^{1}$ A. Oganesian, ${ }^{2}$ S. A. Coats, ${ }^{2}$ C. J. Booth, ${ }^{2}$ C. J. Kros, ${ }^{1}$ \\ R. A. Seifert, ${ }^{2}$ D. F. Bowen-Pope, ${ }^{2}$ and G. P. Richardson ${ }^{1}$ \\ ${ }^{1}$ School of Biological Sciences, University of Sussex, Falmer, Brighton, BN1 9QG, United Kingdom, and 2Department of Pathology, University of \\ Washington, Seattle, Washington 98195
}

\begin{abstract}
A screen for protein tyrosine phosphatases (PTPs) expressed in the chick inner ear yielded a high proportion of clones encoding an avian ortholog of protein tyrosine phosphatase receptor Q (Ptprq), a receptor-like PTP. Ptprq was first identified as a transcript upregulated in rat kidney in response to glomerular nephritis and has recently been shown to be active against inositol phospholipids. An antibody to the intracellular domain of Ptprq, anti-Ptprq, stains hair bundles in mice and chicks. In the chick ear, the distribution of Ptprq is almost identical to that of the $275 \mathrm{kDa}$ hair-cell antigen (HCA), a component of hair-bundle shaft connectors recognized by a monoclonal antibody $(\mathrm{mAb})$ that stains inner-ear hair bundles and kidney glomeruli. Furthermore, anti-Ptprq immunoblots a $275 \mathrm{kDa}$ polypeptide immunoprecipitated by the anti-HCA mAb from the avian inner ear, indicating that the HCA and Ptprq are likely to be the same molecule. In two transgenic mouse strains with different mutations in Ptprq, anti-Ptprq immunoreactivity cannot be detected in the ear. Shaft connectors are absent from mutant vestibular hair bundles, but the stereocilia forming the hair bundle are not splayed, indicating that shaft connectors are not necessary to hold the stereocilia together; however, the mice show rapid postnatal deterioration in cochlear hair-bundle structure, associated with smaller than normal transducer currents with otherwise normal adaptation properties, a progressive loss of basal-coil cochlear hair cells, and deafness. These results reveal that Ptprq is required for formation of the shaft connectors of the hair bundle, the normal maturation of cochlear hair bundles, and the long-term survival of high-frequency auditory hair cells.
\end{abstract}

Key words: hair cell; mechanotransduction; stereocilia; development; inositol phosphatase; podocyte

\section{Introduction}

The sensory epithelia of the inner ear are composed of two types of polarized epithelial cells: hair cells and their surrounding supporting cells. Hair cells have a mechanosensitive hair bundle located at their apical end. The hair bundle is composed of modified microvilli, known as stereocilia, that are arranged in rows of increasing height and coupled to one another by a number of morphologically distinct links: tip links, horizontal top connectors, shaft connectors, and ankle links (Bagger-Sjöbäck and Wersäll, 1973; Pickles et al., 1984; Furness and Hackney, 1985; Csukas et al., 1987; Jacobs and Hudspeth, 1990; Nagel et al., 1991; Goodyear and Richardson, 1992, 1999) [see Goodyear and Richardson (1994) their Fig. 1, for a summary diagram of link distribution on chick hair bundles]. Most hair bundles also possess a single kinocilium located immediately adjacent to the tallest row of stereocilia. A fifth link type, the kinocilial link, connects the kinocilium to its neighboring stereocilia (Hillman, 1969; Bagger-Sjöbäck and

\footnotetext{
Received April 7, 2003; revised Aug. 14, 2003; accepted Aug. 18, 2003.

This work was supported by grants from The Wellcome Trust (057410/Z/99Z) and the National Institutes of Health (DK54857). We thank Jane Bryant, Tao Kwan, and Kala Puligilla for helpful comments on this manuscript, and Sarah Martin for technical assistance.

Correspondence should be addressed to either of the following: Dr. Dan Bowen-Pope, Department of Pathology, University of Washington, Seattle, WA 98195, E-mail: bp@u.washington.edu; or Dr. Guy P. Richardson, School of Biology, University of Sussex, Falmer, Brighton, BN1 9QG, UK, E-mail: g.p.richardson@sussex.ac.uk.

M. B. Wright's present address: F-Hoffmann-La Roche, Basel 4070, Switzerland.

Copyright $\odot 2003$ Society for Neuroscience $\quad 0270-6474 / 03 / 239208-12 \$ 15.00 / 0$
}

Wersäll, 1973; Erneston and Smith, 1986; Nagel et al., 1991; Goodyear and Richardson, 2003). The available evidence indicates that the tip link gates the mechanotransducer channel of the hair cell (Pickles et al., 1984; Assad et al., 1991; Kachar et al., 2000). The functions of the other link types are not known, although they may serve to transmit forces across the hair bundle and maintain its structural integrity.

Little is known about the molecular composition of these various link types. In the chick, they can be distinguished by their relative sensitivities to the calcium chelator BAPTA and the protease subtilisin (Goodyear and Richardson, 1999). Monoclonal antibodies have been raised that recognize epitopes associated with these links in the chick. The $275 \mathrm{kDa}$ hair-cell antigen (HCA) (Richardson et al., 1990) is associated with shaft connectors (Goodyear and Richardson, 1992), the ankle-link antigen (ALA) is a component of ankle links (Goodyear and Richardson, 1999), and the tip-link antigen (TLA) is associated with tip links and kinocilial links (Goodyear and Richardson, 2003). Although these antigens are detected only on hair bundles within the inner ear, the epitopes recognized by the anti-ALA and anti-TLA monoclonal antibodies (mAbs) are also expressed in the retina where they localize to the ciliary calyx of the photoreceptor (Goodyear and Richardson, 1999, 2003), and the HCA is expressed in the kidney where it is associated with the podocytes of the glomeruli (Richardson et al., 1990).

The nonsensory supporting cells of the inner ear have mi- 
crovilli on their apical surface, similar to those found at the apex of other polarized epithelial cell types. A monoclonal antibody recognizing the apical surface of supporting cells and brushborder membranes in the gut and the kidney reacts with a receptor-like protein tyrosine phosphatase (RPTP) known as the supporting-cell antigen (Kruger et al., 1999). During a degenerate RT-PCR screen for other RPTPs expressed in the sensory epithelia of the chick inner ear, a large proportion of the clones isolated were found to encode an avian ortholog of rat Ptprq, an RPTP originally discovered from a transcript upregulated in kidney mesangial cells in response to glomerular nephritis (Wright et el., 1998). Recent studies have shown that Ptprq is highly active against inositol phospholipids (Oganesian et al., 2003). In this study, we reveal that Ptprq is required for shaft-connector formation and the normal maturation of developing cochlear hair bundles, and provide evidence that the HCA is Ptprq.

\section{Materials and Methods}

Degenerate RT-PCR. Degenerate PCR primers based on conserved sequences in the catalytic domain of PTPs were designed according to Norris et al. (1997). Forward primers incorporated BamHI sites, and reverse primers incorporated EcoRI sites. The following forward primers were based on the amino acid consensus sequence FWXMXW: PTPF1, CGGGATCCAYTTYTGGVRRATGRTNTGG; PTPF2, CGGGATCCAYTTYTGGMDKATGRTHTGGSA. Reverse primers based on the amino acid consensus sequence HCSAG were the following: PTPR1, CGGAATTCCNAYDCCHGCRCTRCARTG; PTPR2, CGGAATTCGCCCRAYNGCNGCNCTRCARTG; PTPR3, CGGAATTCCTAGACCNGCRCTRCARTG. Total RNA was isolated from 12 utricles of postnatal day (P) 2 chicks using Trizol (Invitrogen, Paisley, UK). First-strand cDNA was synthesized from $1.0 \mu \mathrm{g}$ of total RNA using $0.5 \mu \mathrm{g}$ of oligo $\mathrm{d}(\mathrm{T})$ primers, $1 \mathrm{~mm} \mathrm{dNTP}$, and $15 \mathrm{U}$ of avian myeloblastosis virus reverse transcriptase (Promega UK, Southampton, UK) in $20 \mu \mathrm{l}$ of $10 \mathrm{~mm}$ Tris$\mathrm{HCl}, \mathrm{pH} 9.1,50 \mathrm{~mm} \mathrm{KCl}, 0.1 \%$ Triton $\mathrm{X}-100$ at $42^{\circ} \mathrm{C}$ for $60 \mathrm{~min}$. The first-strand cDNA was denatured at $98^{\circ} \mathrm{C}$ for $5 \mathrm{~min}$, chilled on ice, and diluted to a final volume of $100 \mu \mathrm{l}$ with water. Aliquots $(5 \mu \mathrm{l})$ of firststrand cDNA were amplified by PCR using TaqEXPRESS (Genetix, New Milton, UK) and the following primer pairs: PTPF1 with PTPR1, PTPF2 with PTPR2, and PTPF1 with PTPR3. Reactions were hot started by adding $50 \mathrm{~mm} \mathrm{MgCl}_{2}$ to a final concentration of $2 \mathrm{~mm}$ and cycled five times at $98^{\circ} \mathrm{C}$ for $15 \mathrm{sec}, 47^{\circ} \mathrm{C}$ for $15 \mathrm{sec}$, and $68^{\circ} \mathrm{C}$ for $30 \mathrm{sec}$, followed by 30 cycles at $98^{\circ} \mathrm{C}$ for $15 \mathrm{sec}, 53^{\circ} \mathrm{C}$ for $15 \mathrm{sec}$, and $68^{\circ} \mathrm{C}$ for $30 \mathrm{sec}$. Reactions were separated on 1\% agarose preparative gels and products of $\sim 400 \mathrm{bp}$ isolated with Geneclean (Anachem, Luton, UK). Products were digested with BamHI and EcoRI and ligated into doubly digested pBluescript SK-. Ligation mixes were transformed into Escherichia coli Xl1-Blue, and recombinant plasmids were identified by blue-white screening. Sixteen white colonies were picked, plasmids were miniprepped, and the DNA sequences of the inserts were determined on both strands. Several hundred of the remaining white colonies were picked into single wells of 96-well plates containing $100 \mu \mathrm{l}$ of Luria-Bertani (LB) medium supplemented with $10 \%$ glycerol. Plates were sealed and incubated overnight with shaking at $37^{\circ} \mathrm{C}$. The 96 -well plates were then replica plated onto LB agar containing $50 \mu \mathrm{g} / \mathrm{ml}$ ampicillin. The 96 -well plates were stored at $-80^{\circ} \mathrm{C}$, and the replica plates were grown overnight at $37^{\circ} \mathrm{C}$. Colony lifts were prepared from the replica plates and probed with ${ }^{32} \mathrm{P}$-labeled inserts pooled from all sequenced plasmids. Colonies that did not hybridize to the mixed probe were identified as novel. They were grown overnight, plasmid DNA was prepared, and DNA sequence was obtained. The similarity of clones to known PTPs was assessed using Basic Local Alignment Search Tool searches (Altschul et al., 1990).

Immunofluorescence microscopy. Tissues were fixed in $3.7 \%$ formaldehyde $/ 0.025 \%$ glutaraldehyde in $0.1 \mathrm{~m}$ sodium phosphate buffer, $\mathrm{pH} 7.4$, for $1 \mathrm{hr}$ at room temperature, washed three times with PBS $(150 \mathrm{~mm}$ $\mathrm{NaCl}, 10$ mm sodium phosphate, $\mathrm{pH} 7.2$ ), equilibrated overnight at $4^{\circ} \mathrm{C}$ with $30 \%$ sucrose in PBS, and imbedded in $1 \%$ low gelling point agarose (type VII; Sigma, Poole, UK) in PBS containing 18\% sucrose. Agarose blocks containing the tissues were rapidly frozen onto cryotome chucks using Cryospray (Bright Instrument, Huntingdon, UK) and sectioned to a thickness of $10 \mu \mathrm{m}$ at a temperature of $-30^{\circ} \mathrm{C}$. Sections were mounted onto gelatin-coated glass microscope slides, dried, and stored at $-20^{\circ} \mathrm{C}$ until use. For immunostaining, cryosections were preblocked with Trisbuffered saline (TBS) (150 mm NaCl, 10 mm Tris-HCl, pH 7.4) containing 10\% heat-inactivated horse serum (TBS/HS) for $1 \mathrm{hr}$, stained overnight with primary antibodies diluted in TBS/HS, and washed three times with TBS. Bound primary antibodies were detected by incubating the sections in FITC-conjugated swine anti-rabbit Ig or FITC-conjugated rabbit anti-mouse Ig followed by FITC-conjugated swine anti-rabbit Ig (Dako, Peterborough, UK) diluted (1:100) in TBS/HS for $1 \mathrm{hr}$. To double label for F-actin, sections were additionally stained with $10 \mathrm{ng} / \mathrm{ml}$ rhodamine-conjugated phalloidin (Sigma). To triple label chick inner ear sections for the HCA, Ptprq, and F-actin, sections were incubated overnight in a mixture of anti-HCA mAb and anti-Ptprq antibody (both at a dilution of 1:100), washed, and labeled with a mixture of FITCconjugated goat anti-mouse IgG (Sigma), rhodamine-conjugated swine anti-rabbit Ig (Dako), and Alexa 350-conjugated phalloidin (Molecular Probes, Cambridge BioSciences, Cambridge, UK), all at a dilution of 1:100. To double label chick kidney section for the HCA and Ptprq, sections were incubated overnight in the same mixture of primary antibodies, followed by Alexa 488-conjugated goat anti-rabbit Ig (1:500 dilution) and rhodamine-conjugated goat anti-mouse IgG (Sigma; 1:200 dilution). Sections were finally washed three times in TBS, mounted in Vectashield (Vector Laboratories, Peterborough, UK), and viewed using either a Zeiss Axioplan 2 microscope equipped with an $100 \mathrm{~W}$ Attoarc fluorescence illuminator or a Zeiss LSM510 confocal microscope. For the confocal color images, the anti-HCA mAb staining was pseudocolored green, the anti-Ptprq antibody staining was red, and the phalloidin staining was blue. The following antibodies and antisera were used in this study: mouse monoclonal anti-HCA D10 (Richardson et el., 1990), mouse monoclonal anti-TLA G19 (Goodyear and Richardson, 2003), rabbit antibodies to the recombinant intracellular domain of rat Ptprq (amino acids R1932 to the C terminus, M2302; GenBank accession number AF063249) (Seifert et al., 2003), and rabbit antibodies to the C-terminal peptide of rat Ptprq (HQPVCFVNYSTLQKMDSLDAMEGD). Rabbit antibodies were affinity purified on their respective antigens.

Phalloidin staining of mouse cochlear whole mounts. Inner ears from embryonic and early postnatal mice were fixed as described above, washed three times in PBS, and dissected to expose the organ of Corti by removing the cartilaginous capsule and the lateral wall of the cochlear duct. The combined greater and lesser epithelial ridges were then detached as a single spiral coil from the central modiolar core of the cochlea and stained with rhodamine-conjugated phalloidin at a concentration of $10 \mathrm{ng} / \mathrm{ml}$ in TBS containing $0.1 \%$ Triton X-100 (TBS/TX) for $30 \mathrm{~min}$ at room temperature. After several washes in TBS/TX, the spirals were separated into an apical and a basal portion, mounted in Vectashield, and viewed with a Zeiss LSM510 confocal microscope using $63 \times$ water immersion lens (numerical aperture 1.2).

Light and electron microscopy. With tissues from mice of $7 \mathrm{~d}$ and older, the inner ears were placed in PBS, small holes were made in the oval and round windows and in the bony shell at the apex of the cochlea, a small amount of fixative $(\sim 50 \mu \mathrm{l})$ was directed through these openings, and the samples were placed in fixative for $2 \mathrm{hr}$. With tissues from younger animals, the entire labyrinth was immersed directly in fixative. For scanning electron microscopy, the tissues were fixed in $2.5 \%$ glutaraldehyde in $0.1 \mathrm{M}$ sodium cacodylate, $\mathrm{pH}$ 7.2. For light and transmission electron microscopy (TEM), labyrinths were fixed in $2.5 \%$ glutaraldehyde in $0.1 \mathrm{M}$ sodium cacodylate, $\mathrm{pH}$ 7.2. In most cases the primary fixative also contained either $1 \%$ tannic acid or $0.5 \%$ ruthenium red. To provide optimal fixation of shaft connectors, utricular maculas were dissected from the labyrinths in HEPES-buffered (10 mM, pH 7.2) HBSS (HBHBSS), the otoconial membrane was carefully removed using fine needles, and the tissue pieces were sucked into a wide-bore plastic pipette tip loaded with fixative containing ruthenium red and transferred to a tube containing the same fixative. After primary fixation, all samples were washed three times in $0.1 \mathrm{M}$ sodium cacodylate buffer and postfixed in $1 \%$ osmium tetroxide, also buffered with $0.1 \mathrm{~m}$ sodium cacodylate for $1 \mathrm{hr}$. For 
samples initially fixed in the presence of ruthenium red, the osmium fixative also contained $0.5 \%$ ruthenium red. After osmication, samples were washed three times with cacodylate buffer, once with $\mathrm{H}_{2} \mathrm{O}$, and dehydrated through a series of ascending concentrations of ethanol. Samples for scanning electron microscopy were critical point dried from liquid $\mathrm{CO}_{2}$, mounted on aluminum stubs using double-sided sticky tape, sputter coated with gold, and viewed in a Leica Leo S420 scanning microscope operating at $20 \mathrm{kV}$. Samples for light and transmission electron microscopy were equilibrated with propylene oxide and imbedded in Epon 812 resin. Blocks were cured for $2 \mathrm{~d}$ at $60^{\circ} \mathrm{C}$ and sectioned with glass and diamond knives. One-micrometer-thick sections for light microscopy were mounted on glass slides, stained with $1 \%$ toluidine blue, and viewed with a Zeiss Axioplan with a $20 \times$ lens. Thin sections $(90-200 \mathrm{~nm}$ thickness) were mounted on copper grids, double stained with uranyl acetate followed by lead citrate, and viewed in a Hitachi transmission electron microscope operating at $75 \mathrm{kV}$.

Immunoprecipitation. Maculas and cristas were dissected from the inner ears of 1-7 d posthatch chicks in Tris-buffered saline or HBHBSS containing a mixture of protease inhibitors [ $1 \mathrm{~mm}$ benzamidine, $1 \mu \mathrm{g} / \mathrm{ml}$ leupeptin, $10 \mu \mathrm{g} / \mathrm{ml}$ pepstatin, $0.1 \mathrm{~mm}$ phenylmethylsulfonylfluoride [(PMSF)], collected into ice-cold buffer containing the same combination of inhibitors with the concentration of leupeptin and PMSF increased to $10 \mu \mathrm{g} / \mathrm{ml}$ and $1.0 \mathrm{~mm}$ respectively, and frozen in liquid $\mathrm{N}_{2}$. Pooled frozen tissue samples (from $\sim 200$ inner ears) were thawed in $\mathrm{PBS} / 1 \%$ Triton X-100 containing the same protease inhibitor mixture used for tissue collection and homogenized in an all-glass, hand-held homogenizer. The homogenate was centrifuged at $39,200 \times g_{\max }$ for 10 min. Aliquots of the supernatant were immunoprecipitated by adding D10 anti-HCA or G19 anti-TLA tissue culture supernatants to a concentration of $1 \%(\mathrm{v} / \mathrm{v})$ and incubating the samples overnight at $4^{\circ} \mathrm{C}$. Rabbit anti-mouse Ig was added to a concentration of $0.5 \mu \mathrm{g} / \mathrm{ml}$, and the immune complexes were collected on Protein A-Sepharose beads. After extensive washing with TBS containing $0.1 \%$ Triton X-100, the beads were heated at $98^{\circ} \mathrm{C}$ in $2 \times$ concentrated SDS-PAGE sample buffer for 4 min. The eluted samples were run on $7.5 \%$ polyacrylamide gels and transferred to PVDF membrane by semidry blotting. Blots were preincubated in TBS containing $0.05 \%$ Tween $20 / 3 \%$ low-fat milk powder for 1 $\mathrm{hr}$ and incubated overnight in affinity-purified rabbit anti-rat Ptprq intracellular domain antibodies $(2.75 \mu \mathrm{g} / \mathrm{ml})$. Bound antibodies were detected with alkaline phosphatase-conjugated goat anti-rabbit Ig.

Transgenic mice. Two regions of the Ptprq gene were replaced independently with the neomycin resistance cassette by homologous recombination in 129/Sv embryonic stem (ES) cells. Correctly targeted ES cell clones were identified by Southern blot analysis using external probes, injected into blastocysts, and reimplanted into pseudopregnant female mice. Chimeras were tested for germ line transmission, and two mouse lines were established and maintained on a mixed C57BL/6J (50\%)/ $129 / \mathrm{Sv}$ (50\%) background. These are referred to as (1) Ptprq-TM-KO, in which a 2396 bp genomic fragment of Ptprq, containing the exon encoding the transmembrane domain of Ptprq and flanking intronic sequences, is replaced by the neomycin resistance cassette, and (2) PtprqCAT-KO, in which a 1061 bp genomic fragment of Ptprq, containing two exons that encode part of the cytoplasmic domain of Ptprq including the catalytic site, as well as flanking intronic sequences, is replaced by the neomycin resistance cassette. Full details of the preparation of these mice are to be published elsewhere (M. B. Wright, S. A. Coats, R. A. Seifert, C. J. Booth, and D. F. Bowen-Pope, unpublished observations).

Genotyping. Routine genotyping of mice in the Ptprq-TM-KO and Ptprq-CAT-KO mutant colonies was performed by PCR. Genomic DNA was isolated from tail snips according to Malumbres et al. (1997). For the Ptprq-TM-KO mutant, aliquots $(2 \mu \mathrm{l})$ of tail DNA were added to $46 \mu \mathrm{l}$ PCR reactions containing 10\% DMSO, $200 \mu \mathrm{M}$ dNTP, $1.25 \mathrm{U}$ of TaqEXPRESS (Genetix Ltd), and 25 pmol each of primers RQgenoF1 (CCAATGGGATACTTGGTCTTCTAGC), RQgenoR1 (TTGCAAGGGATTGCTCAACCATCTC), and PGKR2 (CACGAGACTAGTGAGACGTGCTACT) in $50 \mathrm{~mm}$ Tris- $\mathrm{HCl}, \mathrm{pH}$ $9.1,16 \mathrm{~mm}$ ammonium sulfate, $150 \mu \mathrm{g} / \mathrm{ml}$ BSA. Reactions were heated to $98^{\circ} \mathrm{C}$ for $1 \mathrm{~min}$, hot started by the addition of $2 \mu \mathrm{l}$ of $50 \mathrm{mM} \mathrm{MgCl}_{2}$, and heated to $98^{\circ} \mathrm{C}$ for $15 \mathrm{sec}, 55^{\circ} \mathrm{C}$ for $20 \mathrm{sec}, 68^{\circ} \mathrm{C}$ for $30 \mathrm{sec}$, for $40 \mathrm{cycles}$.
For the Ptprq-CAT-KO mutant, aliquots $(2 \mu \mathrm{l})$ of tail DNA were added to separate PCRs for the wild-type and mutant alleles. The wild-type reactions were as described above except that DMSO was not used and the primers were RQF6 (CTGCTACTGAGCTATAGACAGCCTC) and RQR6 (CAGTCATGCAGTCTCCATGCTAGAC). The mutant allele was amplified with the primers TMKOF (CTGCCGAGAAAGTATCCATCAT) and TMKOR (TTAGGAAAGGACAGTGGGAGTG) in the presence of $10 \%$ DMSO. PCR products were analyzed by $1.5 \%$ agarose $1 \times$ TBE gel electrophoresis. In the Ptprq-TM-KO mice, a single band of $350 \mathrm{bp}$ is amplified from wild types, two products of 350 and $450 \mathrm{bp}$ are amplified from heterozygotes, and a single product of $450 \mathrm{bp}$ is seen in homozygous mutants. In the Ptprq-CAT-KO mice, a single band of 250 bp is amplified from wild types, two products of 250 and $580 \mathrm{bp}$ are amplified from heterozygotes, and a single product of $580 \mathrm{bp}$ is seen in homozygous mutants.

$R T$-PCR. Total RNA was isolated from the cochleas of wild-type or homozygous Ptprq-TM-KO and Ptprq-CAT-KO mice using Trizol reagent (Invitrogen). First-strand cDNA was synthesized from $1 \mu \mathrm{g}$ of total RNA as described above for degenerate RT-PCR, except that $0.5 \mu \mathrm{g}$ of random hexamers was used in place of oligo $\mathrm{d}(\mathrm{T})$ primers, and the reaction was incubated at room temperature for $10 \mathrm{~min}$ followed by $60 \mathrm{~min}$ at $42^{\circ} \mathrm{C}$. Aliquots $(2 \mu \mathrm{l})$ of first-strand cDNA were used for PCR with TMKOF4 (TTACTGACTCTGAGTACTCTGAC) and TMKOR2 (ATCTCTCGTCCTTCAGCTCCAAGTC), to amplify a 261 bp product spanning the exon encoding the TM domain of PTPRQ. In homozygous Ptprq-TM-KO mice, this primer pair will amplify a product of $161 \mathrm{bp}$ that lacks the exon encoding the TM domain. Aliquots of first-strand cDNA were also amplified with CATKOF2 (CGAGGACAACAAGCCAGTGACAGTC) and CATKOR2 (TTCCATGGCATCCAGAGAGTCCATC) to amplify a 508 bp product spanning two exons encoding part of the catalytic domain of PTPRQ. In homozygous Ptprq-CAT-KO mice, this primer pair will amplify a product of $226 \mathrm{bp}$ in which these two exons are missing. All reactions contained $50 \mathrm{pmol}$ of each primer, $200 \mu \mathrm{M}$ dNTP, and 1.25 U TaqDNA polymerase (Bioline, London UK) in $50 \mathrm{~mm}$ Tris- $\mathrm{HCl}, \mathrm{pH}$ 9.1, $16 \mathrm{~mm}$ ammonium sulfate, $150 \mu \mathrm{g} / \mathrm{ml} \mathrm{BSA}$, and were subjected to 40 cycles of $98^{\circ} \mathrm{C}$ for $15 \mathrm{sec}, 52^{\circ} \mathrm{C}$ for $20 \mathrm{sec}, 72^{\circ} \mathrm{C}$ for $50 \mathrm{sec}$. Products were analyzed by $1.5 \%$ agarose gel electrophoresis.

Electrophysiology. Outer hair cells (OHCs) from the apical and basal coils of homozygous Ptprq-TM-KO mutant mice and their heterozygous littermate controls were studied after acute dissection of the organ of Corti (ages P5-P7; day of birth is P0). Tissues from mice used for these experiments were frozen for subsequent genotyping. Extracellular solution was bath applied at a rate of $6 \mathrm{ml} / \mathrm{hr}$ and contained (in $\mathrm{mm}$ ): 135 $\mathrm{NaCl}, 5.8 \mathrm{KCl}, 1.3 \mathrm{CaCl}_{2}, 0.9 \mathrm{MgCl}_{2}, 0.7 \mathrm{NaH}_{2} \mathrm{PO}_{4}, 2 \mathrm{Na}$-pyruvate, 5.6 D-glucose, 10 HEPES-NaOH, pH 7.5, osmolality $\sim 306 \mathrm{mOsm} / \mathrm{kg}$. Amino acids and vitamins for Eagle's MEM were added from concentrates (Invitrogen). OHCs (Ptprq-TM-KO +/-: 11 cells; Ptprq-TM-KO $-/-: 18$ cells) were whole-cell voltage clamped at room temperature $\left(22-25^{\circ} \mathrm{C}\right)$ using an EPC-8 patch-clamp amplifier (HEKA, Lambrecht, Germany). Patch pipettes were pulled from soda glass capillaries and coated with wax. The intracellular solution for recording mechanoelectrical transducer currents contained (in $\mathrm{mm}$ ): $135 \mathrm{CsCl}, 2.5 \mathrm{MgCl}_{2}, 1$ EGTA-CsOH, $2.5 \mathrm{Na}_{2}$ ATP, $10 \mathrm{Na}_{2}$ phosphocreatine, 5 HEPES-CsOH, $\mathrm{pH} 7.3,290 \mathrm{mOsm} / \mathrm{kg}$. In the experiments in which basolateral outward currents were recorded, the intracellular solution contained (in mM): 131 $\mathrm{KCl}, 3 \mathrm{MgCl}_{2}, 1$ EGTA-KOH, $5 \mathrm{Na}_{2} \mathrm{ATP}, 10 \mathrm{Na}_{2}$ phosphocreatine, 5 HEPES-KOH, pH 7.3, $292 \mathrm{mOsm} / \mathrm{kg}$. Data were filtered at $2.5 \mathrm{kHz}$, sampled at $5 \mathrm{kHz}$, and stored on computer for off-line analysis. All membrane potentials were corrected for a $-4 \mathrm{mV}$ liquid junction potential between pipette and bath solutions. Series resistance after electronic compensation of up to $80 \%\left(R_{\mathrm{s}}\right)$ was $2.5 \pm 0.2 \mathrm{M} \Omega(n=29)$, resulting in an average voltage-clamp time constant of $16 \pm 2 \mu \mathrm{sec}$. Correction of membrane potentials for the voltage drop across the series resistance was applied only for $\mathrm{K}^{+}$current analysis, because the drop for the transducer currents was at most $2.7 \mathrm{mV}$. Mechanoelectrical transducer currents were elicited using fluid-jet stimulation $(45 \mathrm{~Hz}$ sinewaves or $50 \mathrm{msec}$ steps filtered at $1 \mathrm{kHz}$; eight-pole Bessel) as described previously (Kros et al., 1992). Bundle movements were recorded with a laser differential interferometer, and bundle stiffness was measured as described previ- 
Table 1. Receptor-like protein tyrosine phosphatases obtained by RT-PCR

\begin{tabular}{lclll}
\hline $\begin{array}{l}\text { Number } \\
\text { of clones }\end{array}$ & $\begin{array}{l}\text { DNA sequence } \\
\text { identity (\%) }\end{array}$ & RPTP & Accession number & $\begin{array}{l}\text { Orthologous mouse } \\
\text { gene symbol }\end{array}$ \\
\hline 16 & 84 & Rn rPTP-GMC1 & NM_022925 & Ptprq \\
12 & 100 & Gg SCA & AJ238216 & N.D. \\
6 & 97 & Gg Cryp $\alpha$ & L32780 & Ptprs \\
6 & 87 & Hs RPTP $\delta$ & NM_130393 & Ptprd \\
4 & 83 & Mm LAR & NM_011213 & Ptprf \\
3 & 97 & Gg PTP $\lambda$ & Z21960 & Ptprc \\
1 & 84 & Mm RPTP- $\kappa$ & NM_008983 & Ptprk \\
1 & 99 & Gg RPTP $\zeta / \beta$ & L27625 & Ptprz1
\end{tabular}

Table summarizing the clones obtained from chick utricular maculas using RT-PCR with degenerate primers fo RPTPs and their identity with known RPTPs. Rn, Rattus norvegicus; Gg, Gallus gallus; Hs, Homo sapiens; Mm, Mus musculus; N.D., no designated symbol.

ously (Kros et al., 1995; Géléoc et al., 1997). Mean data are quoted \pm SEM, and comparisons are based on the unpaired two-tailed $t$ test, with $p<0.05$ as the criterion for statistical significance.

Hearing tests. Visual observation of the Preyer reflex, a back-flick of the pinna in response to sound, was used to assess hearing in mice. The animals were placed in a large, open-top, plastic box, and a $20 \mathrm{kHz}$ tone burst was delivered at intensities of $\sim 90,100$, and $105 \mathrm{~dB}$ sound pressure level from distances of $\sim 30,20$, and $10 \mathrm{~cm}$ above the head, respectively, using a custom-built device provided by the Medical Research Council Institute of Hearing Research (Nottingham, UK).

\section{Results}

Total RNA prepared from the utricular maculas of $2 \mathrm{~d}$ posthatch chicks was amplified by RT-PCR using three pairs of degenerate primers based on conserved sequences in the catalytic domains of known PTPs. Specific products were cloned and sequenced. From a total of 49 clones sequenced that encoded RPTPs (Table 1), approximately one-third (16 of 49 ) had $84 \%$ similarity to an RPTP now known as Ptprq (formerly rPTP-GMC1) that was identified in a differential display screen for transcripts upregulated in response to experimentally induced nephritis in the rat kidney (Wright et al., 1998). Other RPTPs expressed in the utricular maculas were, in order of abundance of clones obtained, the SCA, Cryp- $\alpha$, RPTP- $\delta$, LAR, PTP $\lambda$, RPTP- $\kappa$ and RPTP $\zeta / \beta$ (Table 1).

Ptprq is a type III RPTP, with an extracellular domain containing 18 fibronectin (FN) type III repeats, a short hydrophobic membrane-spanning region, and an intracellular domain containing a single consensus PTPase catalytic site (Wright et al., 1998). Recent studies have shown that Ptprq is a phosphatidylinositol phosphatase (Oganesian et al., 2003). Antibodies to the intracellular domain of recombinant Ptprq (Seifert et al., 2003) were used to determine the localization of this enzyme in the avian and mammalian inner ear. In sections of the early posthatch chick inner ear, anti-Ptprq specifically stains the hair bundles of the sensory hair cells, in a manner that is almost, but not strictly, identical to that observed with the anti-HCA mAb D10 (Fig. $1 a-j$ ). By double labeling with phalloidin it can be seen that the anti-HCA mAb and the anti-Ptprq antibody stain the hair bundle up to its distal tip in the extrastriolar regions of the macula (Fig. $1 a-c$ ), whereas they only stain the basal region of the hair bundle in the basilar papilla (Fig. $1 f-h$ ) and the striolar regions of the maculas (data not shown). Two rabbit sera raised to the predicted C-terminal peptide of Ptprq stain hair bundles in the avian inner ear in a manner similar to that observed with the antibody raised to the recombinant intracellular domain (data not shown). Although the staining patterns observed with the anti-Ptprq antibodies are similar to those described previously for the antiHCA mAb (Richardson et al., 1990) and correlate with the known
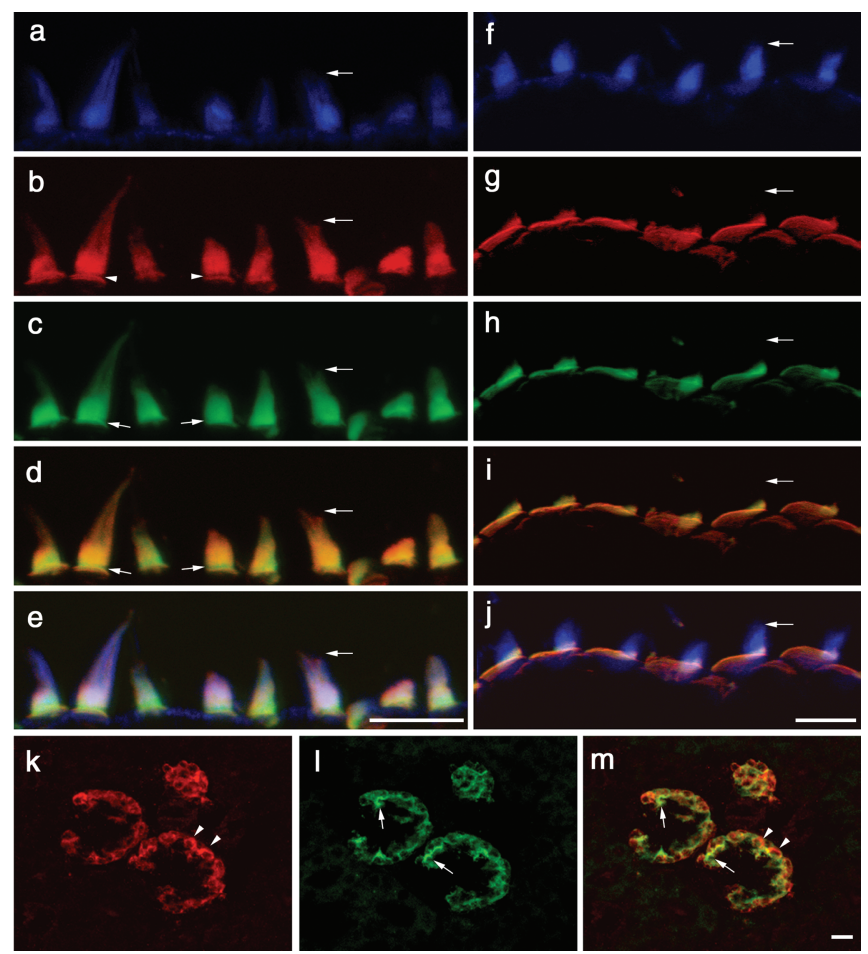

Figure 1. Distribution of anti-HCA and anti-Ptprq immunostaining in the inner ear and kidney of the early posthatch chick. $a-j$, Compressed z-stacks of confocal sections from the extrastriolar region of the utricular macula $(a-e)$ and the basilar papilla $(f-j)$, triple-labeled with Alexa 350 phalloidin $(a, f)$, polyclonal anti-Ptprq $(b, g)$, and monoclonal anti-HCA mAb $(c$, $h$ ). $d, i$, Merges of anti-Ptprq and anti-HCA mAb staining; $e, j$, merges of the images obtained through all three channels (anti-Ptprq, anti-HCA mAb, and F-actin). Horizontal arrows point to the tip of the same hair bundle in $a-e$ and $f-j$. $b$, Arrowheads indicate the ankle-link region that is not stained by anti-Ptprq. $c, d$, Small arrows indicate that anti-HCA mAb staining is present in the ankle-link region. $b-d$, In the extrastriolar region of the macula, the hair bundle is stained to its distal tip by anti-Ptprq and the anti-HCA mAb. $g-i$, In the basilar papilla, staining with both antibodies is restricted to the proximal end of the hair bundle. $g, h$, The extensive, nonstereociliary apical membrane of the hair cell is clearly stained by both antibodies in the basilar papilla. $k, l$, Compressed z-stacks of confocal section from the chick kidney double labeled with anti$\operatorname{Ptprq}(k)$ and the anti-HCA mAb ( $/)$. m, Merge of anti-Ptprq and anti-HCA mAb staining. Arrowheads indicate areas that stain only with anti-Ptprq; arrows indicate areas that stain only with the anti-HCA mAb. Scale bars, $10 \mu \mathrm{m}$.

distribution of shaft connector material on the apical membrane of the hair cell (Goodyear and Richardson, 1992), merged overlays indicate subtle differences in the staining patterns observed with the two antibodies (Fig. 1d,i). On extrastriolar vestibular hair cells, a narrow region around the base of the hair bundle fails to label with the polyclonal antibody against the intracellular domain of Ptprq (Fig. 1b), and over the entire lower region of the bundle, the intensity of staining observed with the anti-HCA $\mathrm{mAb}$ is generally greater than that observed with anti-Ptprq (Fig. 1c). On hair cells from the basilar papillas and striolar region of the maculas, both antibodies stain the base of the hair bundle and the apical nonstereociliary surface of the hair cell (Fig. $1 g, h$ ), but anti-Ptprq antibody stains the base of the hair bundle weakly relative to the apical nonstereociliary surface (Fig. $1 g$ ). The inverse is observed with the anti-HCA mAb, with the base of the hair bundle staining more intensely than the apical, nonstereociliary surface (Fig. 1h). As a consequence of these differences, the color of the merged images is not uniform across the top end of the hair cells (Figs. 1d,i). In the kidney, anti-Ptprq and the antiHCA mAb both stain glomeruli (Fig. $1 k, l$ ), and although there are clearly large areas of overlap, there are also regions that stain 
with anti-Ptprq and not with the antiHCA $m A b$ and vice versa (Fig. $1 \mathrm{~m}$ ).

In the inner ear of the early postnatal mouse, the anti-Ptprq antibody stains hair bundles in the cochlea and in the vestibule (Fig. $2 a-c$ ). Staining is restricted to the hair bundles and not detected in any other cell type within the inner ear. As in the chick with the anti-HCA mAb, Ptprq distribution on hair bundles differs according to the type of hair cell or its location (Fig. $3 a-d$ ). With mature inner hair cells in the cochlea (Fig. $3 b, b^{\prime}$ ), and with vestibular hair cells in the striolar region of the macula (Fig. $3 c, c^{\prime}$ ) and at the crest of the crista (Fig. $3 d, d^{\prime}$ ), double labeling with phalloidin and anti-Ptprq (Fig. $3 a-d$ ) reveals that Ptprq is restricted to the basal region of the hair bundle. With extrastriolar hair cells (Fig. 3c, $c^{\prime}$ ) and those around the base of the crista (Fig. $3 d, d^{\prime}$ ), hair bundles are stained up to their distal tips by anti-Ptprq. Staining is not observed in mouse kidney with anti-Ptprq (data not shown), although it does stain podocytes in the glomerulus of the human kidney (Seifert et al., 2003). The anti-HCA mAb D-10 is specific for avian tissue and does not cross-react with hair cells in the mammalian inner ear.

These results suggested that the HCA may be Ptprq, or closely associated with it. Triton X-100 soluble extracts prepared from the sensory epithelia of the avian inner ear therefore were immunoprecipitated with the anti-HCA mAb or an irrelevant mAb of the same isotype, and the immunoprecipitates were immunoblotted with the polyclonal anti-Ptprq antibody. A band with a molecular mass of $\sim 275 \mathrm{kDa}$ is recognized by anti-Ptprq in samples immunoprecipitated with the anti-HCA mAb, but not in the control samples (Fig. 4).

The HCA is expressed early during the development of the avian inner ear, coincident with the onset of hair-bundle appearance (Bartolami et al., 1991). We therefore examined the appearance of the Ptprq in the developing mouse inner ear (Fig. 5). Ptprq is first detected in the vestibule at embryonic day (E) 13.5 (Fig. $5 a, a^{\prime}$ ). This is coincident with the onset of hair-cell differentiation, as judged by myosin VIIA expression, although only a proportion of the myosin VIIA-expressing hair cells express Ptprq at this early stage (Fig. $5 a, a^{\prime}$ ). By E15.5, all myosin VIIApositive hair cells in the vestibule express Ptprq. In the cochlea, the expression of Ptprq is clearly delayed relative to onset of haircell differentiation. Ptprq is first detected on inner hair cells in the basal coil of the cochlea at E17.5, $2 \mathrm{~d}$ after the appearance of myosin VIIa positive hair cells in this region at E15.5 (data not shown). Outer hair cells in the basal end of the cochlea begin to express Ptprq $1 \mathrm{~d}$ after the inner hair cells, on E18.5 (Fig. 5b, $b^{\prime}$ ). Staining is weak at this stage but becomes stronger by P2 (Fig. $\left.5 c, c^{\prime}\right)$. In the very apical end of the cochlea, expression of Ptprq in both inner and outer hair cells is not observed until P2. Ptprq is expressed only transiently by basal-coil outer hair cells during their development. A decrease in hair-bundle staining for Ptprq on outer hair cells becomes apparent at P15 in the basal end of the cochlea (data not shown). By P21, Ptprq is no longer detected on basal-coil outer hair cells (Fig. $5 d, d^{\prime}$ ). In contrast, the hair bundles of inner hair cells (Fig. $5 d, d^{\prime}$ ), apical-coil outer hair cells (data not shown), and those in the vestibular system (Fig. $5 e, e^{\prime}$ ) continue to express Ptprq at high levels until at least 6 months of age (the oldest stage studied).

RT-PCR analysis of total RNA prepared from the cochleas of Ptprq-TM-KO and Ptprq-CAT-KO mice indicates that mRNA transcripts spanning the deleted exons are expressed in mice ho- mozygous for these deletions (Fig. 6). However, Ptprq protein cannot be detected by immunofluorescence microscopy in the inner ears of mice that are homozygous for these mutations (Fig. $7 a-f$ ). In mice that are heterozygous for these mutations (Fig. $7 b, e)$, the level and distribution of immunostaining are similar to those seen in wild-type mice (Fig. $7 a, d$ ). Hypothetically, exon skipping could lead to the production of truncated forms of Ptprq in these mice. In Ptprq-CAT-KO mice, a catalytically inactive form with a shortened intracellular domain and a wild-type $\mathrm{C}$ terminus could be produced; in Ptprq-TM-KO mice a nonmembrane-bound form comprising only the extracellular domain could be produced. Both of these potential variants might act as dominant-negative forms of the enzyme. Antisera raised to the $\mathrm{C}$ terminus of Ptprq do not stain hair cells in PtprqCAT-KO mice (data not shown), indicating that the predicted protein product is unstable and that the mouse is a functional null. Antibodies are not available that recognize the extracellular domain of mouse Ptprq, so the possibility that an extracellular domain variant of the protein is produced in the Ptprq-TM-KO mice cannot be formally excluded; however, an identical phenotype is observed in both the Ptprq-TM-KO and the PtprqCAT-KO mice, strongly suggesting that both are functional null mutants in which any protein produced is unstable.

Mice that are homozygous for either deletion, Ptprq-TM-KO or Ptprq-CAT-KO, fail to respond with a Preyer reflex to a $20 \mathrm{kHz}$ tone burst at 3 months of age. Light microscopy was used to examine the morphology of the cochlea (Fig. $8 a-d$ ) and vestibular organs (Fig. 8e,f) at this stage of development. The gross structure of the organ of Corti in the apical, low-frequency end of the cochlea in wild-type (data not shown), heterozygous (Fig. 8a), and homozygous (Fig. 8b) Ptprq-CAT-KO mice is similar. In the basal, high-frequency region of wild-type (data not shown) and heterozygous (Fig. 8c) Ptprq-CAT-KO mice, the organ of Corti also appears structurally normal. In homozygous PtprqCAT-KO mutant mice (Fig. $8 d$ ), however, there is an absence of hair cells and, in some animals, a complete loss of the organ of Corti. Identical observations were made in Ptprq-TM-KO mice. A loss of hair cells is not observed in any of the vestibular organs in homozygous Ptprq-CAT-KO (Fig. 8e,f) or Ptprq-TM-KO (data not shown) mice.

Using phalloidin staining, defects in hair-bundle morphology are observed in homozygous Ptprq-TM-KO and Ptprq-CAT-KO mice by P1, in the basal-most regions of the cochlea (Fig. 9). At E18.5, hair-bundle structure in a wild-type and homozygous mutant appears similar (Fig. 9a,b). At P1, a phenotype is observed most readily in the inner hair cells (Fig. $9 c, d$ ). The rows of stereocilia in the hair bundles of wild-type or heterozygous hair cells are very tightly defined (Fig. $9 c$ ), whereas hair cell bundles of homozygous mutant hair cells show varying degrees of disorganization (Fig. 9d). By P8, defects in hair-bundle structure in homozygous mutants become more apparent and are readily evident on both inner and outer hair cells (Fig. 10a-d). The same phenotype is observed in Ptprq-CAT-KO (Fig. 10a,b) and Ptprq- 

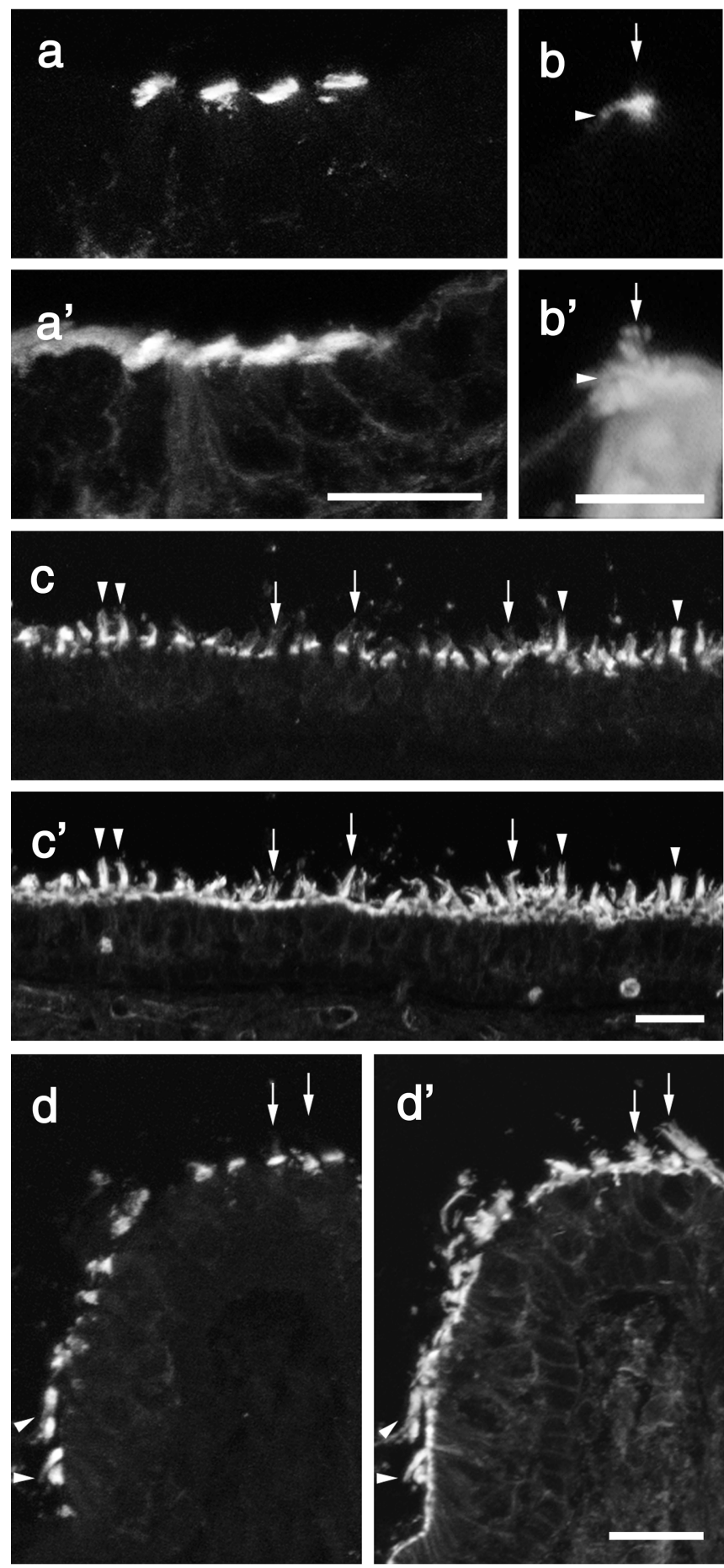

Figure 3. Fluorescence micrographs from mouse inner ear sections double labeled with affinity-purified antibodies to $\operatorname{Ptprq}(a-d)$ and rhodamine phalloidin $\left(a^{\prime}-d^{\prime}\right)$. On cochlear inner hair cells $\left(b, b^{\prime}\right.$, arrows) and hair cells in the striolar regions of the utricular macula $\left(c, c^{\prime}\right.$, arrows) and at the apex of the crista $\left(d, d^{\prime}\right.$, arrows), anti-Ptprq staining is concentrated at the proximal end of the hair bundle. With hair bundles in the extrastriolar regions of the utricular macula ( $c$, $c^{\prime}$, arrowheads) and peripheral region of the crista $\left(d, d^{\prime}\right.$, arrowheads), the hair bundle is stained up to its tip. Sections are from mice at P2 $(a), \mathrm{P} 21(b, c)$, and P15 (d). Scale bars: $a, 20$ $\mu \mathrm{m} ; b, 10 \mu \mathrm{m} ; c, d, 20 \mu \mathrm{m}$.

TM-KO (Fig. 10c,d) mice. On homozygous mutant inner hair cells, many stereocilia are missing, and the remaining stereocilia are often misaligned or fused together (Figs. 10b, $d, 11 a, b$ ). Disruption in the organization and a loss of stereocilia are also observed with the hair bundles of mutant outer hair cells, but ste-

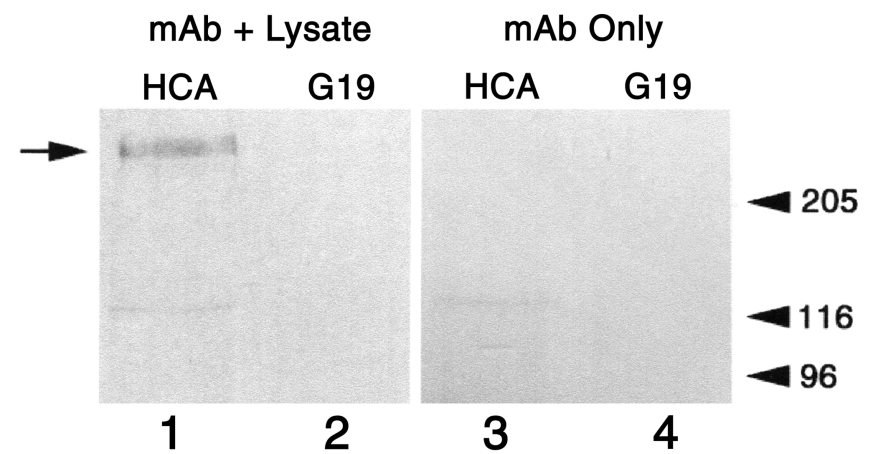

Figure 4. Immunoprecipitation of the HCA. An immunoblot stained with affinity-purified antibodies to the recombinant intracellular domain of Ptprq. Lysates prepared from the sensory epithelia of the chick inner ear (lanes 1,2) or lysis buffer (lanes 3, 4) were incubated with an anti-HCA mAb (mAb D10; lanes 1,3) or an irrelevant $m A b$ of the same isotype ( $m A b$ G19; lanes $2,4)$, and the mAbs were precipitated with rabbit anti-mouse Ig and collected with Protein A-Sepharose. The $275 \mathrm{kDa}$ hair-cell antigen is indicated by the arrow on the left. Markers indicated on the right with molecular masses in kilodaltons are skeletal muscle myosin (205), $\beta$-galactosidase (116), and phosphorylase B (96)

reocilial fusion is rarely encountered (Figs. 10b,d, 11c,d). The hair bundles of outer hair cells in homozygous mutants are smaller than those of wild-type or heterozygous mice and have a less distinctive $\mathrm{V}$-shaped form and are more U-shaped. In the basal end of the cochlea the hair bundles do not appear to develop the broader, flatter, more wide-angled appearance characteristic of hair cells in this high-frequency region of the cochlea (Fig. 10ad). The phenotype is initially restricted to the hair bundle because the hair cells in homozygous mutants appear to remain otherwise normal, as judged by light microscopy, until at least P22 (Fig. $10 e, f)$, a stage at which defects in hair-bundle structure are very apparent. The cellular processes that lead to the loss of hair cells and the complete disappearance of the organ of Corti between P21 and P80 have not yet been characterized.

Shaft connectors cannot be readily distinguished on cochlear hair cells in early postnatal (P0-P15) mice because of the extensive cell coat that is transiently found on developing hair bundles (R. J. Goodyear, W. Marcotti, C. J. Kros, and G. P. Richardson, unpublished observations). We therefore looked for the presence of shaft connectors and examined their structure in hair cells of the mature (P21) vestibular system. In hair bundles from wildtype and heterozygous animals that have been fixed in the presence of ruthenium red, shaft connectors appear as dense spherical particles, $\sim 20 \mathrm{~nm}$ in diameter, that are suspended between the membranes of adjacent stereocilia by a number of fine, filamentous stalks. These stalks project from the opposing membranes and can be of variable length (Fig. 12a-d). Such particles are also found attached via filamentous stalks to a single membrane, rather than being suspended between two, on the external, outwardly facing surfaces of the stereocilia and on the apical, nonstereociliary surface of the hair cell (Fig. 12a). Shaft connectors, i.e., defined as dense particles plus stalks, are completely absent from the vestibular hair bundles of both Ptprq-TM-KO (data not shown) and Ptprq-CAT-KO mice (Fig. 12e). In these ruthenium red-stained preparations, the stereocilia within the bundles are packed together in an abnormally tight manner, with the opposing membranes of adjacent stereocilia lying in such close proximity that they often appear to be fused to one another. In material fixed in the absence of any contrasting reagent (Fig. 12f,g) or in the presence of tannic acid (data not shown), such tight packing is not observed, and the spacing of stereocilia appears similar 

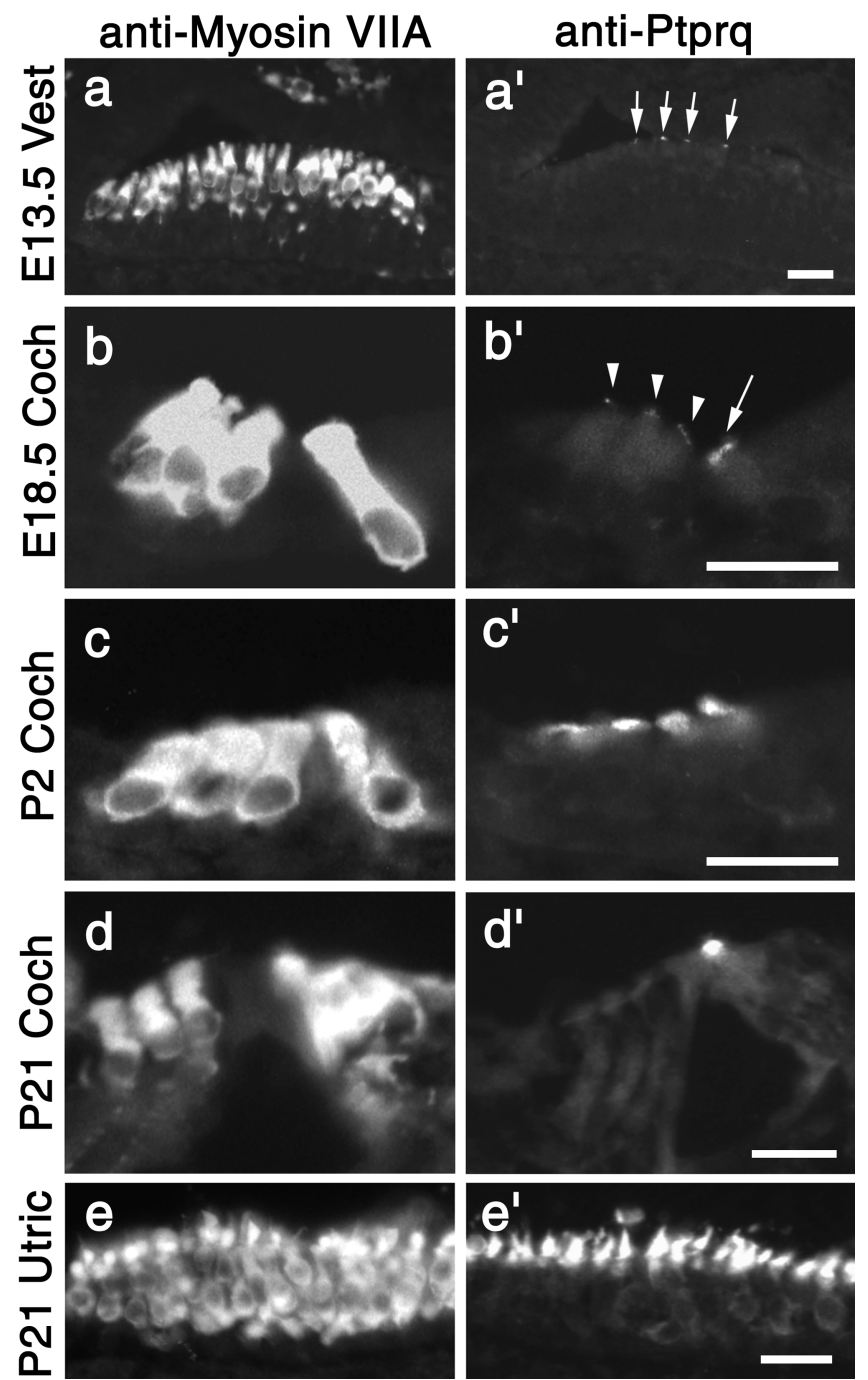

Figure 5. Expression of Ptprq during mouse inner ear development. Pairs of fluorescence micrographs from adjacent sections of the vestibule at E13.5 $\left(a, a^{\prime}\right)$ and P21 $\left(e, e^{\prime}\right)$ and the cochlea at E18.5 $\left(b, b^{\prime}\right)$, P2 $\left(c, c^{\prime}\right)$, and P21 $\left(d, d^{\prime}\right)$ labeled with antibodies to myosin VIlla $(a-e)$ or Ptprq $\left(a^{\prime}-e^{\prime}\right)$. Ptprq first appears in the vestibule at E13.5 $\left(a^{\prime}\right)$ and is present on inner and outer hair cells in the base of the cochlea by E18.5 $\left(b, b^{\prime}\right)$. Ptprq expression on basal-coil outer hair cells increases by P2 $\left(c, c^{\prime}\right)$ but declines by P15 and can no longer be detected at P21 $\left(d^{\prime}\right)$. Inner hair cells $\left(d^{\prime}\right)$ and vestibular hair cells ( $\left.e^{\prime}\right)$ express Ptprq into maturity. Scale bars, $20 \mu \mathrm{m}$.

in heterozygous and homozygous Ptprq mutant hair cells (Fig. $12 f, g)$. Although shaft connectors are completely absent from Ptprq mutant hair cells, a few dense particles could be found just around the base of these hair bundles lying in close proximity to the stereocilial plasma membranes in ruthenium red-contrasted specimens (Fig. 12e).

Electrophysiological recordings were obtained from apicaland basal-coil OHCs of heterozygous and homozygous PtprqTM-KO mice, aged from P5 to P7. The characteristics of the outward $\mathrm{K}^{+}$current, $I_{\mathrm{K}, \text { neo }}$, are similar in heterozygous and homozygous mice (data not shown) and similar to those observed previously in OHCs of wild-type CD1 mice of the same age (Marcotti and Kros, 1999). No significant differences were observed in the activation and reversal potential of the outward $\mathrm{K}^{+}$current. The resting membrane potential of the OHCs was $-65.3 \pm 0.7$ $\mathrm{mV}(n=3)$ in heterozygous control mice and $-64.3 \pm 3.0 \mathrm{mV}$ $(n=4)$ in homozygous mutant mice; the linear leak conductance was $1.6 \pm 0.3 \mathrm{nS}(n=3)$ and $1.3 \pm 0.1 \mathrm{nS}(n=5)$, respectively.

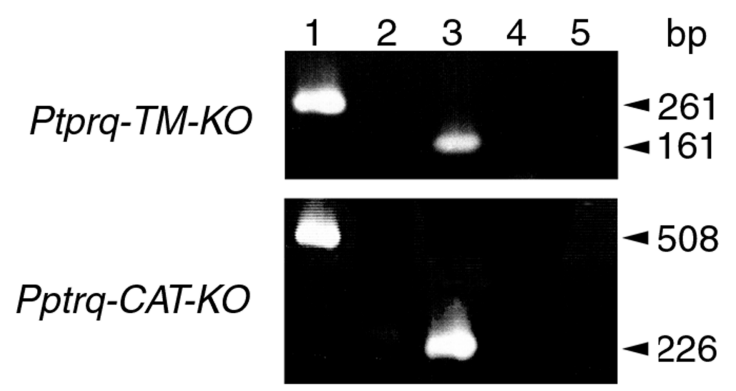

Figure 6. RT-PCR analysis of Ptprq mRNA expression in the cochlea of wild-type, Ptprq-TMKO, and Ptprq-CAT-KO mice. Specific products spanning the exons deleted in the Ptprq-TM-KO and Ptprq-CAT-KO mutants were amplified from RT-PCR reactions performed on total RNA isolated from cochleas from wild-type (lanes 1,2) and homozygous mutant (lanes 3,4) mice. In the Ptprq-TM-K0 line a product of $261 \mathrm{bp}$ is amplified from wild-type mice (lane 1), and a product of $161 \mathrm{bp}$ is amplified from homozygous mutant mice (lane 3). In the Ptprq-CAT-KO line a product of $508 \mathrm{bp}$ is amplified from wild-type mice (lane 1), and a product of $226 \mathrm{bp}$ is amplified from homozygous mutant mice (lane 3). Control reverse transcription reactions performed without reverse transcriptase are negative (lanes 2,4) as are the water controls (lane 5).

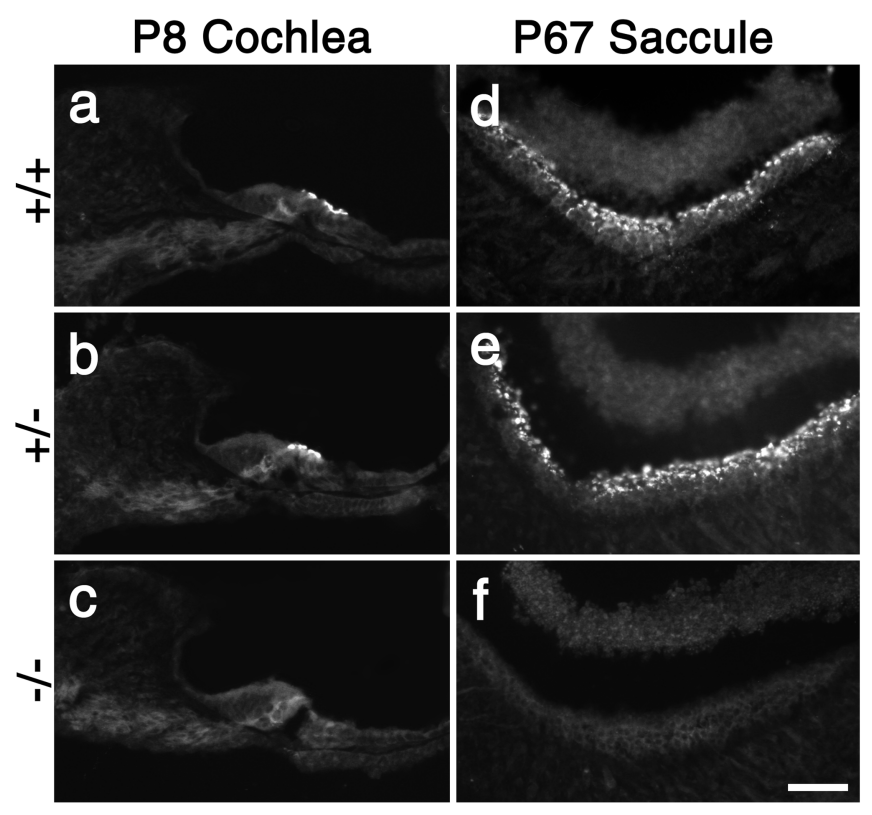

Figure 7. Immunostaining with Ptprq intracellular domain antibodies in the cochlea of wildtype, Ptprq-TM-KO, and Ptprq-CAT-KO mice. Fluorescence micrographs of sections from the cochleas at P8 $(a-c)$ and saccule at P67 $(d-f)$ of wild-type $(a, d)$, heterozygous $(b, e)$, and homozygous $(c, f)$ Ptprq-TM-KO $(a-c)$ and Ptprq-CAT-KO (d-f) mice stained with affinitypurified antibodies to Ptprq. Ptprq cannot be detected with these antibodies in the inner ear of either homozygous mutant $(c, f)$. Scale bar, $50 \mu \mathrm{m}$.

When an oscillating fluid jet is directed at the hair bundle, mechanoelectrical transducer currents can be recorded from the OHCs of both heterozygous (Fig. 13a) and homozygous (Fig. 13b) Ptprq-TM-KO mice. At a holding potential of $-104 \mathrm{mV}$, the currents recorded from the homozygous Ptprq-TM-KO mice $(-467 \pm 55 \mathrm{pA} ; n=10 ; \mathrm{P} 5-\mathrm{P} 7)$ are significantly smaller in amplitude $(p<0.0001)$ than those recorded from the heterozygous Ptprq-TM-KO mice $(-1033 \pm 92$ pA; $n=6$; P5-P7). The shape of the transducer currents in heterozygous and mutant OHCs is similar, and also similar to those recorded previously from wildtype CD1 mice (Kros et al., 1992). When the hair bundles are deflected in the inhibitory direction (negative driver voltage), the transducer current that is partially open at rest is shut off. The size 


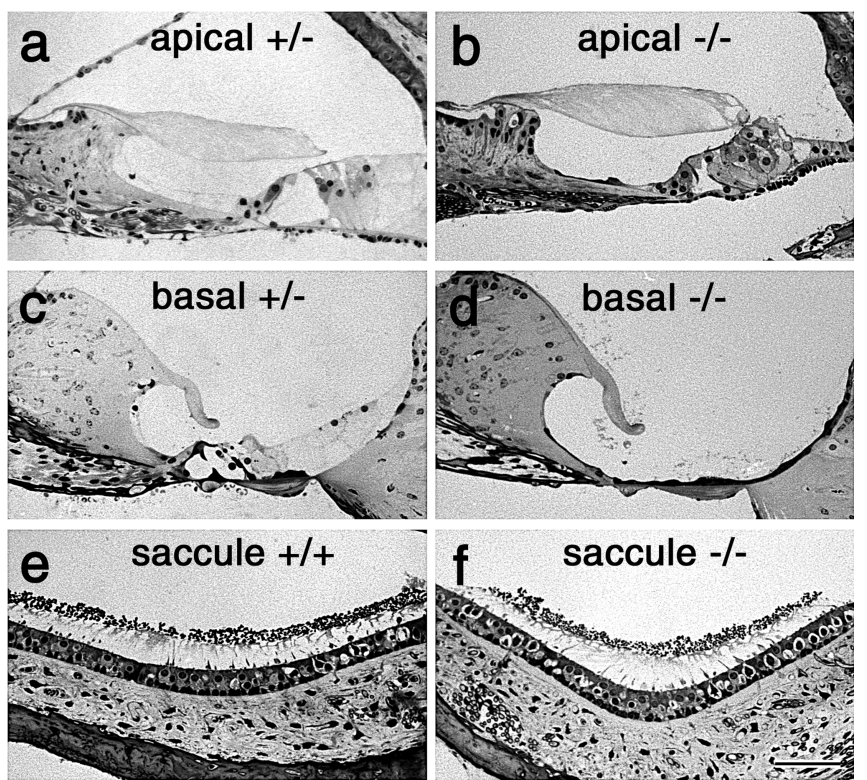

Figure 8. Structure of cochlea in wild-type, Ptprq-TM-KO, and Ptprq-CAT KO mice. Light micrographs of $1-\mu \mathrm{m}$-thick, toluidine blue-stained sections from the apical $(a, b)$ and basal $(c, d)$ regions of the cochlea, and the saccule $(e, f)$, of heterozygous $(a, c)$, wild-type (e), and homozygous ( $b$, $d, f)$ 3-month-old Ptprq-CAT-KO mice. Note the complete loss of the organ of Corti in the basal end of the cochlea in the homozygous Ptprq-CAT-KO ( $d$ ) mouse. Scale bar, $50 \mu \mathrm{m}$.
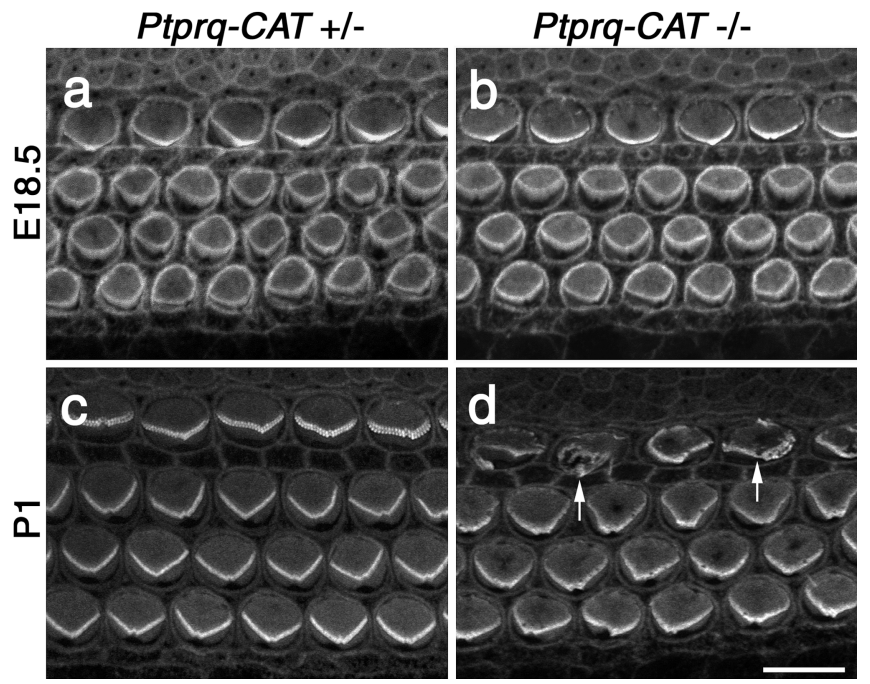

Figure 9. Phalloidin staining of hair bundles in Ptprq-CAT-KO mice. Confocal micrographs of the basal end of phalloidin-stained cochlear whole mounts from heterozygous $(a, c)$ and homozygous Ptprq-CAT-KO $(b, d)$ mice at E18.5 $(a, b)$ and P1 $(c, d)$. Defects in hair-bundle structure are first apparent in the inner hair cells ( $d$, arrows) at P1. Scale bar, $10 \mu \mathrm{m}$.

of this resting current is larger at depolarized potentials $(+96$ $\mathrm{mV}$ ) than at hyperpolarized potentials $(-104 \mathrm{mV})$.

When the bundles of heterozygous and homozygous OHCs are stimulated with force steps, the rapid onset of the transducer current elicited by excitatory stimuli at a holding potential of -84 $\mathrm{mV}$ is followed by a decline (Fig. $13 c, d$, top panels) indicative of adaptation as described for the hair cells of lower vertebrates (Eatock et al., 1987; Howard and Hudspeth, 1987; Assad et al., 1989; Crawford et al., 1989). Two time constants were used to fit onset adaptation (Wu et al., 1999) in mutants and in heterozygous littermate controls. Both the fast and the slow time constant in control OHCs $(0.5 \pm 0.1 \mathrm{msec}$ contributing $69 \%$ of the total

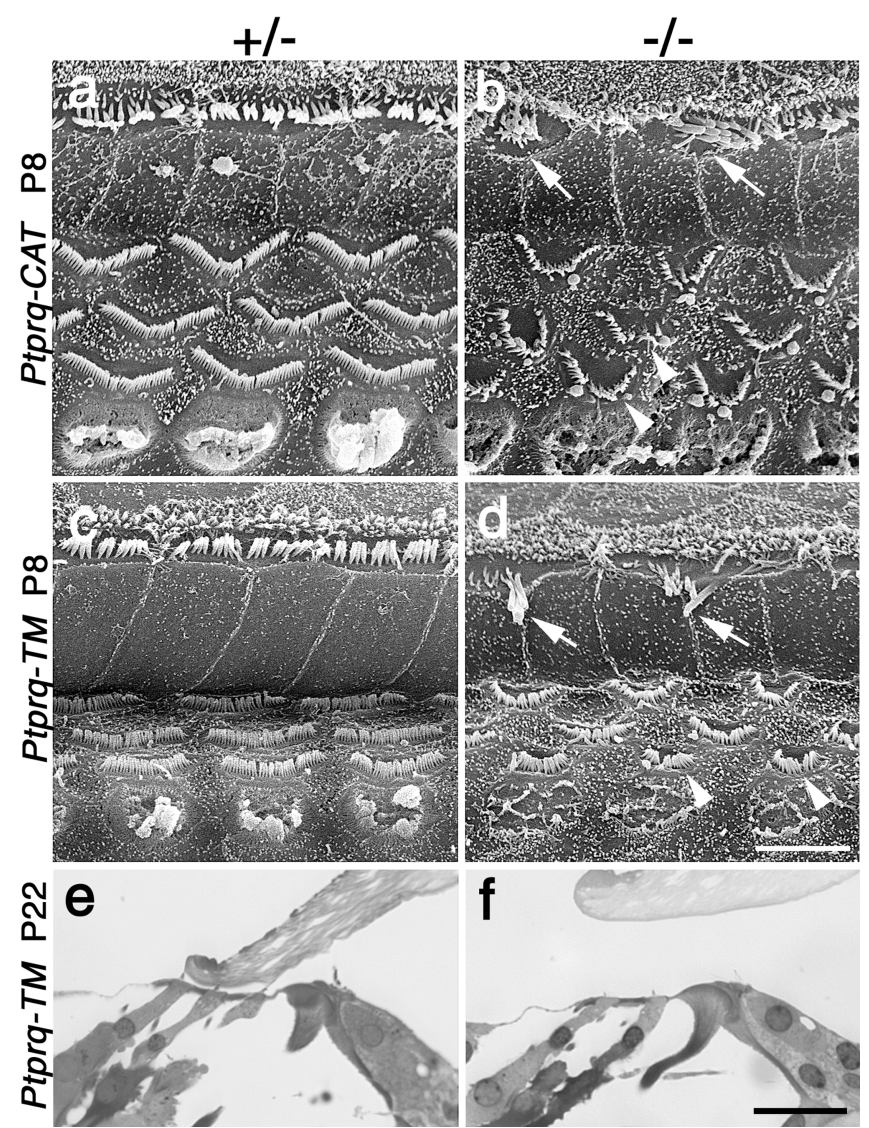

Figure 10. Morphology of hair bundles and hair cells in Ptprq-TM-KO and Ptprq-CAT-KO mice. $a-f$, Survey scanning electron micrographs at P8 $(a-d)$ and sections at P22 $(e, f)$ of the organ of Corti in the basal coil of heterozygous $(a, c, e)$ and homozygous $(b, d, f)$ Ptprq-CAT-KO $(a, b)$ and Ptprq-TM-KO (c-f) mice. The hair bundles of inner (arrows) and outer (arrowheads) hair cells are considerably affected in both the homozygous Ptprq-CAT-KO $(b)$ and the PtprqTM-KO ( $d$ ) mice by P8. Note the difference in the shape of the outer hair-cell hair bundles. Hair cells are still present in the organ of Corti in Ptprq-TM-KO mice at P22 (e, f). Scale bars: (in $d$ ) $a-d, 5 \mu \mathrm{m}$; (in $f) e, f, 20 \mu \mathrm{m}$.

adaptation and $16 \pm 2 \mathrm{msec}$ contributing 31\%; $n=5$ ) are not significantly different from those measured in homozygous mutants $(0.5 \pm 0.2 \mathrm{msec}$ contributing $66 \%$ and $16 \pm 3$ msec contributing $34 \% ; n=5)$. During inhibitory stimuli the resting current is shut off, but a transient rebound (a downward dip in the current trace) is observed at the offset of the inhibitory steps (Fig. $13 c, d$, bottom panels). The maximum size of the rebound is smaller in mutant OHCs $(-106 \pm 29 \mathrm{pA} ; n=6)$ than in heterozygous OHCs $(-218 \pm 65 \mathrm{pA} ; n=7)$, but when expressed as a fraction of the maximum transducer current it is not different between the two groups $(0.30 \pm 0.06$ for both $)$. The rebound adaptation also shows a fast and a slow time constant that did not vary significantly between control $(0.4 \pm 0.1 \mathrm{msec}$ contributing $85 \%$ and $16 \pm 2 \mathrm{msec}$ contributing $15 \% ; n=5)$ and mutant $(0.3 \pm 0.1 \mathrm{msec}$ contributing $80 \%$ and $12 \pm 1 \mathrm{msec}$ contributing $20 \% ; n=4$ ) OHCs. All of these manifestations of adaptation are abolished by stepping the membrane potential to positive values (Fig. 13e,f, top panels, excitatory bundle displacement; bottom panels, inhibitory displacement) as observed previously in hair cells from CD-1 mice (Kros et al., 1992, 1995) and in other vertebrates (Assad et al., 1989; Crawford et al., 1989). The relation between bundle displacement and transducer current is similar for the heterozygous and homozygous hair cells of Ptprq-TM-KO mice (Fig. $13 g, h$ ). After stepping the membrane potential from 

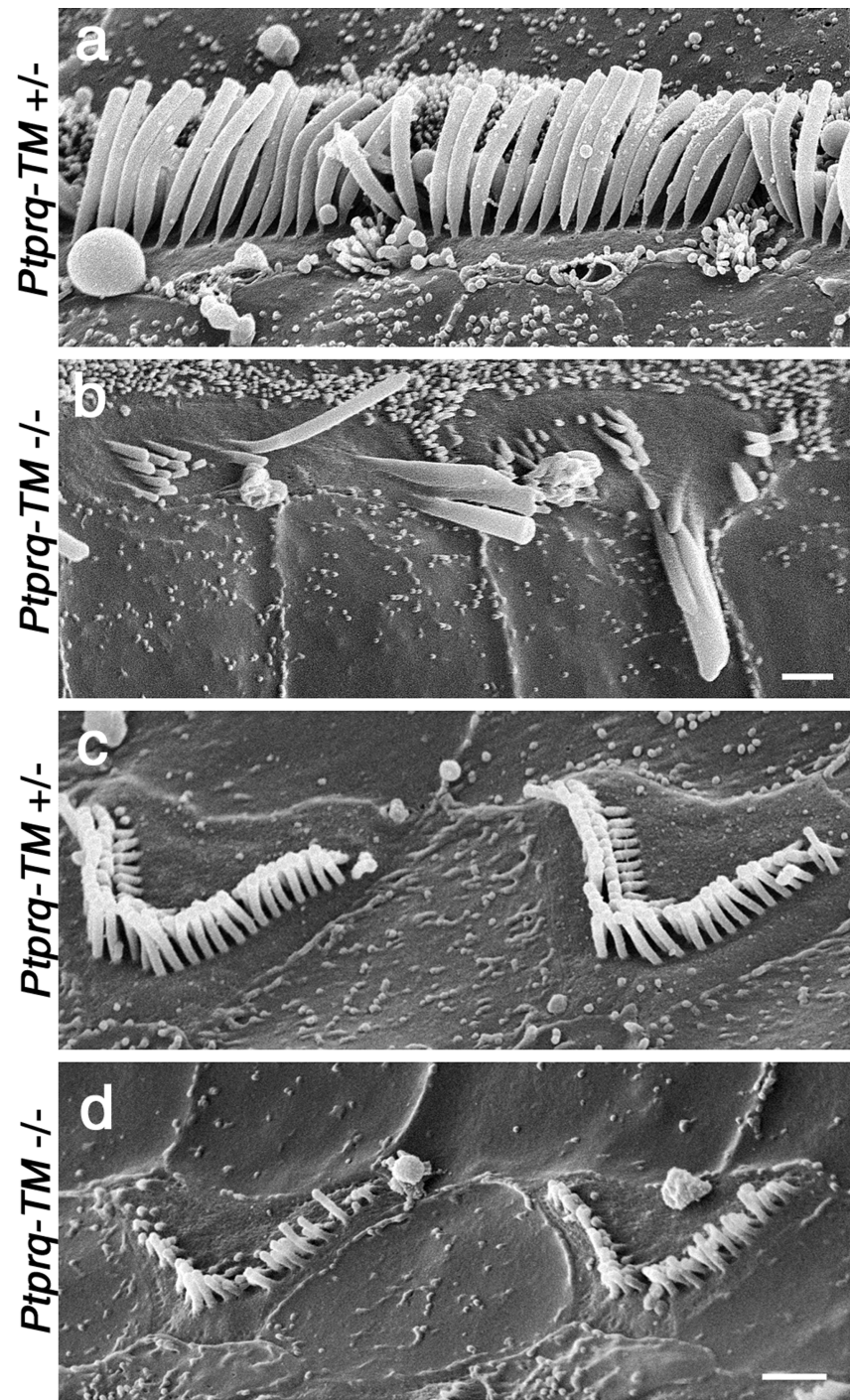

Figure 11. Structure of hair bundles in heterozygous and homozygous Ptprq-TM-KO mice. High-magnification scanning electron micrographs of the hair bundles of inner $(a$, $b)$ and outer $(c, d)$ hair cells in the organ of Corti (basal coil) of heterozygous $(a, c)$ and homozygous $(b, d)$ Ptprq-TM-K0 mice at P15. Note the loss of stereocilia from the hair bundles of homozygous mutant hair cells, the giant, fused stereocilia on the homozygous mutant inner hair cells $(b)$, and how the stereocilia are shorter on the homozygous mutant outer hair cells $(d)$. Scale bars, $1 \mu \mathrm{m}$.

-84 to $+86 \mathrm{mV}$, the resting open probability of the transducer channels increases from 5.4 to $29.2 \%(n=7)$ in heterozygous OHCs and from 7.7 to $34.2 \%(n=5)$ in homozygous mutant $\mathrm{OHCs}$ (not significantly different between homozygotes and heterozygotes), indicative of a reduction in adaptation even in the unstimulated bundle during depolarization. The apparent overall steady-state stiffness of the homozygous $\mathrm{OHC}$ (P7) bundles $(5.4 \pm 0.7 \mathrm{mN} / \mathrm{m} ; n=8)$ was smaller compared with that measured in littermate $(\mathrm{P} 7)$ controls $(6.7 \pm 0.8 \mathrm{mN} / \mathrm{m} ; n=8)$, but the difference is not significant.

\section{Discussion}

The results of this study show that Ptprq, a receptor-like phosphatase (Wright et al., 1998) recently shown to be active against inositol phospholipids (Oganesian et al., 2003), is required for the formation of shaft connectors and the normal maturation of developing hair bundles in the mammalian cochlea. Although

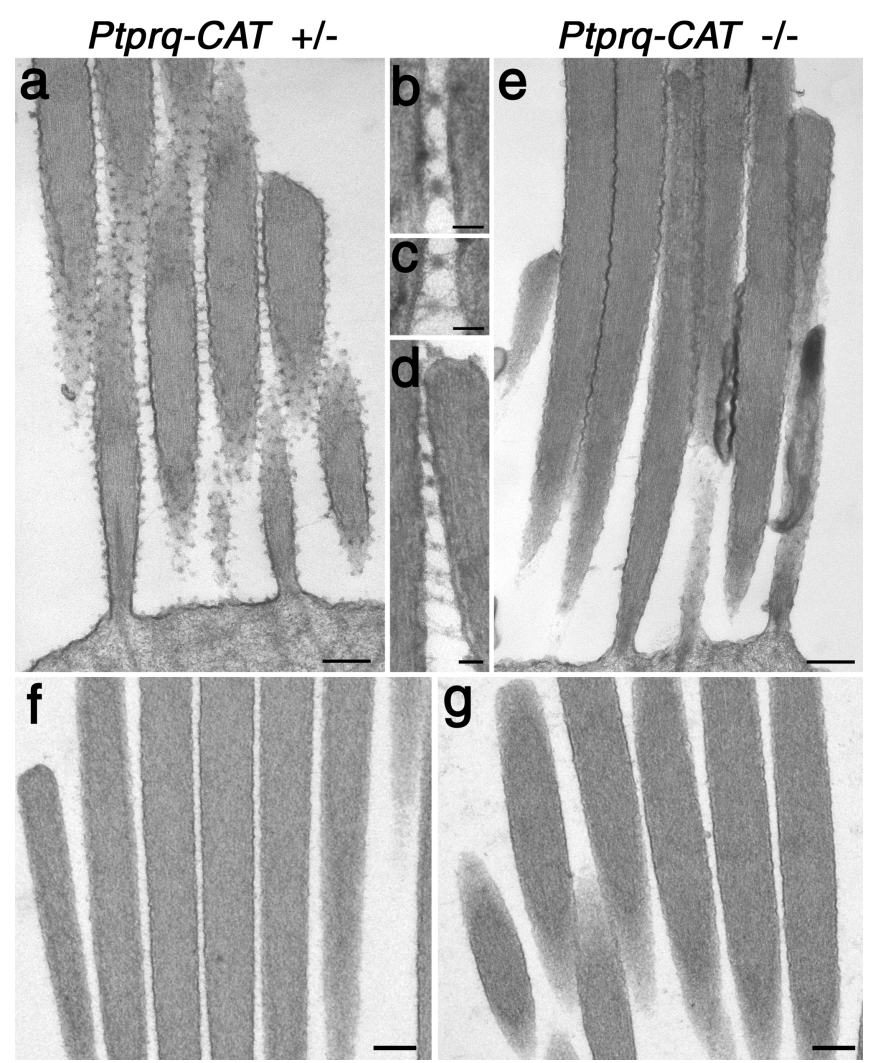

Figure 12. TEM analysis of shaft connector structure in vestibular hair bundles of wild-type and Ptprq-CAT-KO mice. Transmission electron micrographs of extrastriolar hair bundles from the utricular macula of heterozygous $(a-d, f)$ and homozygous $(e, g)$ mutant Ptprq-CAT-KO mice at P21. Samples in $a-e$ were fixed in fixatives containing ruthenium red; samples in $f$ and $g$ were fixed in the absence of any contrasting agents. Note the lack of shaft connectors in the homozygous mutant hair bundle $(e)$ and the close apposition of the stereocilia. Details of shaft connector structure in heterozygous Ptprq-CAT-KO mice $(b-d)$ reveal dense particles attached to the membrane by fine stalks. The spacing of stereocilia in the hair bundles of heterozygous $(f)$ and homozygous $(g)$ mutant Ptprq-CAT-KO mice is similar in the absence of ruthenium red. Scale bars: $a, e-g, 200 \mathrm{~nm} ; b-d, 50 \mathrm{~nm}$.

Ptprq immunoreactivity is detected in human podocytes (Seifert et al., 2003), it cannot be detected in the mouse kidney, and thus far kidney structure and function appear to be completely normal in the Ptprq-KO mice (Booth, Wright, Coats, Seifert, BowenPope, unpublished observations). This study therefore provides the first demonstration of an essential function for Ptprq in vivo. The data also provide evidence suggesting that the HCA is Ptprq.

In the developing vestibule of the mouse, Ptprq appears when hair bundles first emerge, at E13.5 (Denman-Johnson and Forge, 1999); however, there is a delay between the stage at which hair bundles first emerge in the mouse cochlea, (E15.5) (Anniko, 1983; Boeda et al., 2002) and the time when the Ptprq can be first detected (E17.5). This implies that Ptprq is not essential for the early stages of hair-bundle formation in the mouse cochlea. Surprisingly, Ptprq is expressed only transiently by high-frequency, basal-coil OHCs during late embryonic and early postnatal development, over a period of $\sim 3$ weeks. Persistent expression of Ptprq is therefore not normally necessary for the maintenance of hair-bundle structure in mature basal-coil outer hair cells. Nonetheless, the data from Ptprq mutant mice clearly indicate that the transient expression of Ptprq during development is required for the complete maturation of hair-bundle structure in this type of hair cell.

Shaft connectors, a link type described previously on the hair 
PTPRQ-TM (+/-)

a

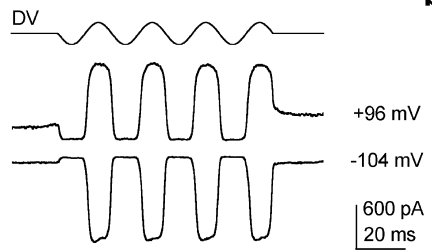

C
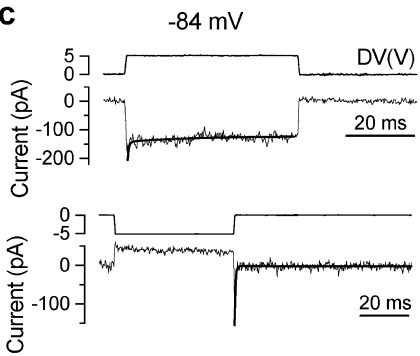

e
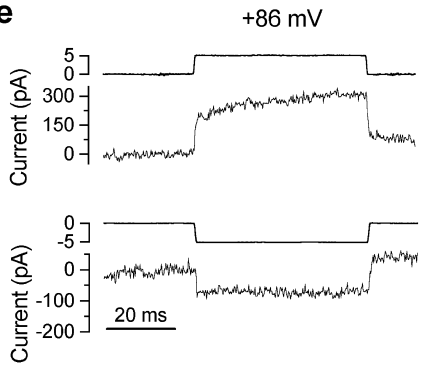

g

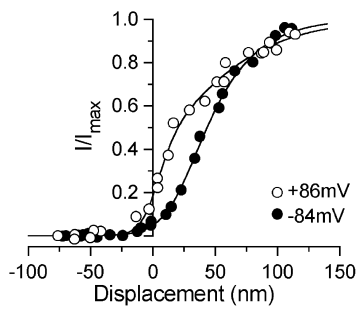

PTPRQ-TM (-/-)

b
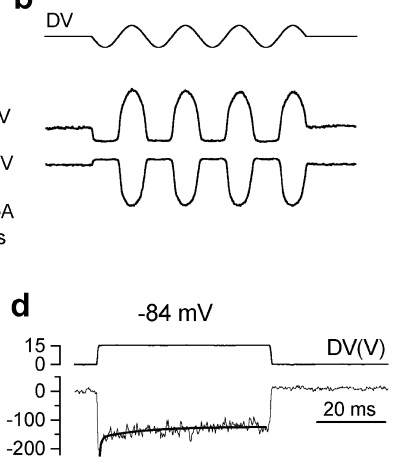

f
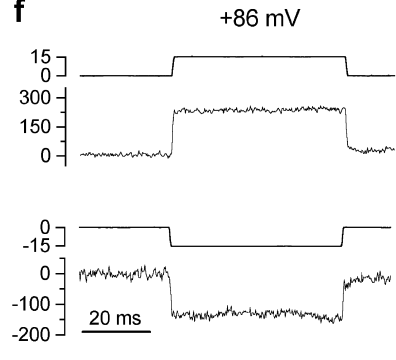

h
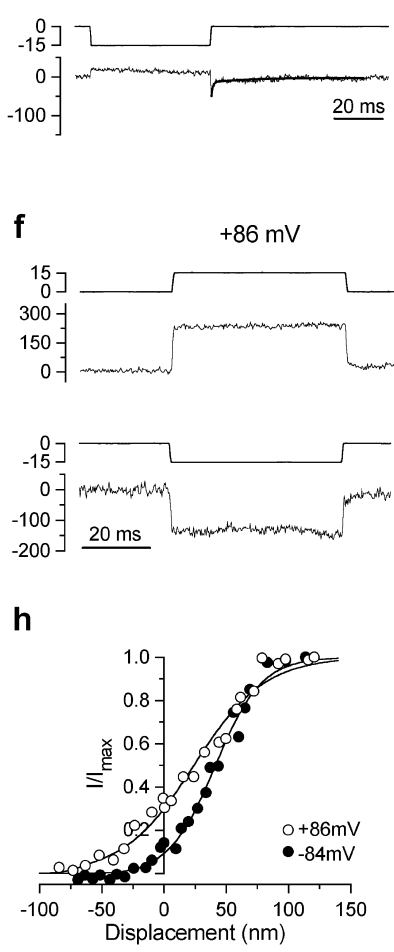

Figure 13. Mechanotransducer currents in Ptprq-TM-KO outer hair cells. Transducer currents recorded in basal-coil OHCs from a heterozygous Ptprq-TM-KO mouse $(a, c, e, g)$ and a homozygous Ptprq-TM-KO mouse $(b, d, f, h) . a, b$, Transducer currents recorded at a holding potential of -104 and $+96 \mathrm{mV}$ by applying sinusoidal force stimuli of $45 \mathrm{~Hz}$. The driver voltage signal (DV) (amplitude, $35 \mathrm{~V}$ ) to the fluid jet is shown above each trace. Positive deflections are excitatory. $c, d$, The time course of onset adaptation to an excitatory step displacement at -84 $\mathrm{mV}$ (top panels). Note the difference in the driver voltages applied. Fitted time constants for onset adaptation are as follows: c, 0.34 and $16.1 \mathrm{msec} ; d, 0.39$ and $12.3 \mathrm{msec}$. Bottom panels show the transducer current in response to a negative stimulus at $-84 \mathrm{mV}$. After termination of the inhibitory stimulus the transducer current shows evidence of rebound adaptation. Fitted time constants for the rebound adaptation are as follows: $c, 0.34$ and $16.2 \mathrm{msec} ; d, 0.54$ and 16.3 msec. $e, f$, The time course of the transducer current elicited by excitatory (top panels) and inhibitory (bottom panels) bundle displacements at $+86 \mathrm{mV}$. Note the absence of onset and rebound adaptation. $a, c, e, P 70 \mathrm{HC}: C_{\mathrm{m}} 6.9 \mathrm{pF} ; R_{\mathrm{s}} 2.4 \mathrm{M} \Omega ; b, d, f, \mathrm{P} 70 \mathrm{OHC}: C_{\mathrm{m}} 6.7 \mathrm{pF} ; R_{\mathrm{s}} 3.0 \mathrm{M} \Omega$. All responses shown are single traces. $g, h$, Normalized peak transducer current as a function of bundle displacement. Zero current is set as the holding current when the force stimulus closes most transducer channels. Smooth curves are second-order Boltzmann functions: $I=I_{\max } /(1$ $\left.+\exp \left(a_{2}\left(x_{2}-x\right)\right)^{*}\left(1+\exp \left(a_{1}\left(x_{1}-x\right)\right)\right)\right) \cdot g_{1}$ At $-84 \mathrm{mV}, I_{\max }=-732 \mathrm{pA}, a_{1}=0.06$ $\mathrm{nm}^{-1}, a_{2}=0.04 \mathrm{~nm}^{-1}, x_{1}=26 \mathrm{~nm}, x_{2}=35 \mathrm{~nm} ;$ at $+86 \mathrm{mV}, I_{\max }=810 \mathrm{pA}, a_{1}=0.12$ $\mathrm{nm}^{-1}, a_{2}=0.02 \mathrm{~nm}^{-1}, x_{1}=7 \mathrm{~nm}, x_{2}=14 \mathrm{~nm}$. P7 0HC: $C_{\mathrm{m}} 7.0 \mathrm{pF} ; R_{\mathrm{s}} 1.4 \mathrm{M} \Omega$. Bundle height $4 \mu \mathrm{m}$; bundle width $8 \mu \mathrm{m}$. $h$, At $-84 \mathrm{mV}, I_{\max }=-211 \mathrm{pA}, a_{1}=0.10 \mathrm{~nm}^{-1}, a_{2}=$ $0.054 \mathrm{~nm}^{-1}, x_{1}=-32 \mathrm{~nm}, x_{2}=41 \mathrm{~nm} ; \mathrm{at}+86 \mathrm{mV}, I_{\max }=316 \mathrm{pA}, a_{1}=0.15 \mathrm{~nm}^{-1}, a_{2}=$ $0.035 \mathrm{~nm}^{-1}, x_{1}=-76 \mathrm{~nm}, x_{2}=24 \mathrm{~nm}$. P7 OHC: $C_{\mathrm{m}} 6.8 \mathrm{pF} ; R_{\mathrm{s}} 2.5 \mathrm{M} \Omega$. Bundle height 3.5 $\mu \mathrm{m}$; bundle width $6 \mu \mathrm{m}$. bundles of fishes, frogs, and chicks (Neugebauer and Thurm, 1985; Nagel et al., 1991; Goodyear and Richardson, 1992), are also found on mouse hair bundles but are completely absent from the hair bundles of homozygous mutant Ptprq-TM-KO and homozygous mutant Ptprq-CAT-KO mice. Each shaft connector is composed of a ruthenium red-reactive, electron-dense particle that is connected to the plasma membrane by several fine strands of variable length. If the $18 \mathrm{FN}$ type III repeats of Ptprq were arranged in an extended, linear conformation, the extracellular domain would be long enough $(\sim 65 \mathrm{~nm})$ to account for the longest of these fine strands. Each strand therefore may represent a single Ptprq ectodomain, and the dense particle may be a region in which several molecules either are interacting homophilically in cis or in trans, or both, or are interacting with an unknown ligand. The reactivity of this particle with ruthenium red suggests that it contains glycosaminoglycans. A recent study has shown that the heparan sulfate proteoglycans agrin and collagen type XVIII are ligands for the type II RPTP Cryp- $\alpha$ (cPTP $\sigma)$ (Aricescu et al., 2002). Furthermore, an $\mathrm{mAb}$ recognizing keratan sulfate stains hair bundles in the guinea pig cochlea (Katori et al., 1996), and a polyclonal antiserum raised against $\alpha$-tectorin, a sulfated, keratanase-sensitive component of the tectorial membrane, stains both the tectorial membrane and hair bundles in the mouse inner ear (Killick and Richardson, 1997). Thus the particle associated with the shaft connector may be a keratan sulfate proteoglycan.

In the chick, the $275 \mathrm{kDa}$ HCA is a component of the shaft connectors (Goodyear and Richardson, 1992). A full-length cDNA for Ptprq cloned from rat kidney (Wright et al., 1998) predicts a polypeptide with a mass of $256,823 \mathrm{Da}$ and 39 potential sites for N-glycosylation (Wright et al., 1998). Native full-length Ptprq is therefore likely to have a mass similar to the mass of 275 $\mathrm{kDa}$ determined for the native chick HCA (Richardson et al., 1990). A polypeptide with a mass similar to that reported for the HCA reacts with the anti-Ptprq antibodies when they are used to immunoblot samples that have been immunoprecipitated from detergent-soluble extracts of the chick inner ear by the anti-HCA $\mathrm{mAb}$. Although this is good evidence that the HCA and Ptprq are one and the same thing, it cannot be entirely excluded that they are two different proteins of very similar mass that interact to form a shaft connector complex. The subtle differences observed in the staining patterns found with anti-Ptprq and the anti-HCA $\mathrm{mAb}$ could be used to support the suggestion that Ptprq and the HCA are different entities. In the mouse, however, it is known that a combination of splicing and the use of an alternative promoter generates three Ptprq isoforms, a full-length form complete with an ectodomain and intracellular cytoplasmic domain, a form with a membrane-tethered ectodomain lacking a catalytic intracellular domain, and a nonmembrane-tethered cytoplasmic form (Seifert et al., 2003). The anti-HCA mAb reacts with an external epitope on the surface of the hair bundle and could react with the first two isoforms, whereas anti-Ptprq will not recognize the second isoform that lacks an intracellular domain. The subtle difference in the staining patterns observed in hair cells with these two antibodies may reflect differences in the relative distribution of these isoforms. For example, the ankle link region of extrastriolar hair cells may contain only the isoform lacking the intracellular domain, thus staining with the anti-HCA mAb and not with anti-Ptprq.

The term "shaft connector" (Neugebauer and Thurm, 1985) implies that these structures act to hold the stereocilia together. Although some RPTPs can act as cell adhesion molecules (BradyKalnay and Tonks, 1995), there is so far no evidence that the type 
III RPTPs have a similar function. Despite a complete absence of shaft connectors from the vestibular hair bundles of the Ptprq mutant mice, there is no indication that the stereocilia are splayed, even in the extrastriolar regions of the maculas where shaft connectors are distributed extensively over the entire apical surface of the hair cell. Although it is always possible that tip links or other unidentified structures can maintain hair-bundle integrity in Ptprq-KO mice, shaft connectors may serve some other function, perhaps acting as spacers. The tight packing of stereocilia seen in ruthenium red-stained Ptprq mutant hair bundles indicates that shaft connectors can prevent stereocilia membranes from coming into close apposition and fusing; however, close membrane apposition is not seen in Ptprq mutant hair bundles when they are fixed in fixatives that do not contain ruthenium red, indicating that shaft connectors are not normally required to maintain spacing.

If Ptprq is neither a connector nor a spacer, what then is its function? Also, why is it required for the normal maturation of cochlear hair bundles and not those in the vestibular system, and why does its loss lead to the preferential loss of basal-coil cochlear hair cells? Ptprq is not required for mechanotransduction. There is no overt, behavioral vestibular phenotype in Ptprq mutant mice, and the OHCs in these mutant mice transduce and have a normal physiological profile despite the appearance of a hairbundle phenotype. The reduction in transducer current amplitude observed in Ptprq-KO mice is most likely caused by the observed loss of stereocilia. In all other respects transduction appears normal in the mutants. Fast, submillisecond adaptation and slow adaptation (Wu et al., 1999), at both the onset of excitatory and the offset of inhibitory stimuli, and the resting transducer current at hyperpolarized and depolarized potentials, are similar to those recorded from heterozygous littermates and those recorded previously in OHCs (Kros et al., 2002). Ptprq is thus unlikely to be involved in transducer current adaptation, either in the fast adaptation process proposed to occur at the level of the transducer channels (Wu et al., 1999; Kennedy et al., 2003) or in the slower adaptation probably involving myosin motors (Holt and Corey, 2000). As a membrane-associated inositol lipid phosphatase (Oganesian et al., 2003), Ptprq is ideally positioned to regulate the local phosphoinositide phospholipid content of the apical membrane of the hair cell. Inositol phospholipids are key regulators of the actin cytoskeleton and membrane traffic (Takenawa and Itoh, 2001), and the rates of membrane and actin turnover (Schneider et al., 2002) in developing cochlear hair bundles may be critically regulated by Ptprq. Differences in susceptibility to Ptprq loss in different types of hair cells may be explained by redundancy; another phosphatase may be expressed in vestibular and apical-coil cochlear hair cells that compensates, either completely or partially, for loss of Ptprq. The preferential loss of hair cells from the basal end of the cochlea may be a secondary consequence of the severity of the hair-bundle phenotype seen in basal-coil cochlear hair cells; the phenotype may eventually become so severe that it completely prevents the hair cells from transducing, resulting in their demise.

Ptprq now joins a growing list of molecules, including the unconventional myosins (myosins VI, VIIa, and XV), cadherin23, protocadherin-15, integrin $\alpha 8 \beta 1$, espin, mucolipin-3, and Tmie1 (Avraham et al., 1995; Probst et al., 1998; Self et al., 1998; Littlewood Evans and Muller, 2000; Zheng et al., 2000; Alagraman et al., 2001; DiPalma et al., 2001, 2002; Mitchem et al., 2002), that are required for the development and maintenance of hairbundle structure. As such, the gene for Ptprq, which is located at the distal end of mouse chromosome 10 and on human 12q15 (Wright et al., 1998), can be considered a potential deafness gene.

\section{References}

Alagraman KN, Murcia CL, Kwon HY, Pawlowski KS, Wright CG, Woychik RP (2001) The mouse Ames waltzer hearing-loss mutant is caused by a mutation of Pdch15, a novel protocadherin gene. Nat Genet 27:99-102.

Altschul F, Gish W, Miller W, Myers EW, Lipman DJ (1990) Basic local alignment research tool. J Mol Biol 215:403-410.

Anniko M (1983) Cytodifferentiation of cochlear hair cells. Am J Otolaryngol 4:375-388.

Aricescu AR, McKinnell IW, Halfter W, Stoker AW (2002) Heparan sulphate proteoglycans are ligands for receptor protein tyrosine phosphatase sigma. Mol Cell Biol 22:1881-1892.

Assad JA, Hacohen N, Corey DP (1989) Voltage dependence of adaptation and active bundle movement in bullfrog saccular hair cells. Proc Natl Acad Sci USA 86:2918-2922.

Assad JA, Shepherd GMG, Corey DP (1991) Tip-link integrity and mechanical transduction in vertebrate hair cells. Neuron 7:987-994.

Avraham KB, Hasson T, Steel KP, Kingsley DM, Russell LB, Mooseker MS, Copeland NG, Jenkins NA (1995) The mouse Snell's waltzer deafness gene encodes an unconventional myosin required for structural integrity of the inner ear hair cells. Nat Genet 11:369-375.

Bagger-Sjöbäck D, Wersäll J (1973) The sensory hairs and the tectorial membrane of the basilar papilla in the lizard Calotes versicolor. J Neurocytol 2:329-350.

Bartolami S, Goodyear R, Richardson G (1991) Appearance and distribution of the $275 \mathrm{kDa}$ hair-cell antigen during development of the avian inner ear. J Comp Neurol 314:777-788.

Boeda B, El-Amraoui A, Bahloul A, Goodyear R, Daviet L, Blanchard S, Perfettini I, Fath KR, Shorte S, Reiners J, Houddusse A, Legrain P, Wolfrum U, Richardson G, Petit C (2002) Myosin VIIA, harmonin, and cadherin 23, three Usher I gene products that cooperate to shape the sensory hair bundle. EMBO J 21:6689-6699.

Brady-Kalnay SM, Tonks NK (1995) Protein tyrosine phosphatases as adhesion receptors. Curr Biol 7:650-657.

Csukas SR, Rosenquist TH, Mulroy MJ (1987) Connections between stereocilia in auditory hair cells of the alligator lizard. Hear Res 30:147-156.

Crawford AC, Evans MG, Fettiplace R (1989) Activation and adaptation of transducer currents in turtle hair cells. J Physiol (Lond) 419:405-434.

Denman-Johnson K, Forge A (1999) Establishment of hair bundle polarity and orientation in the developing vestibular system of the mouse. J Neurocytol 28:821-835.

Di Palma F, Holme RH, Bryda EC, Belyantseva IA, Pellegrino R, Kachar B, Steel KP, Noben-Trauth K (2001) Mutations in Cdh23, encoding a new type of cadherin, cause stereocilia disorganization in waltzer, the mouse model for Usher syndrome type 1D. Nat Genet 27:103-107.

Di Palma F, Belyantseva IA, Kim HJ, Vogt TF, Kachar B, Noben-Trauth K (2002) Mutations in Mcol3 associated with deafness and pigmentation defects in varitint-waddler (Va) mice. Proc Natl Acad Sci USA 99:14994-14999.

Eatock RA, Corey DP, Hudspeth AJ (1987) Adaptation of mechanoelectrical transduction in hair cells of the bullfrog's sacculus. J Neurosci 7:2821-2836.

Erneston S, Smith CA (1986) Stereo-kinociliar bonds in mammalian vestibular organs. Acta Otolaryngol 101:395-402.

Furness DN, Hackney CM (1985) Cross-links between stereocilia in the guinea pig cochlea. Hear Res 18:177-188.

Géléoc GSG, Lennan GWT, Richardson GP, Kros CJ (1997) A quantitative comparison of mechanoelectrical transduction in vestibular and auditory hair cells of neonatal mice. Proc R Soc Lond B Biol Sci 264:611-621.

Goodyear R, Richardson G (1992) Distribution of the 275 kD hair cell antigen and cell surface specialisations on auditory and vestibular hair bundles in the chicken inner ear. J Comp Neurol 325:243-256.

Goodyear R, Richardson G (1994) Differential glycosylation of auditory and vestibular hair bundle proteins revealed by peanut agglutinin. J Comp Neurol 345:267-278.

Goodyear R, Richardson G (1999) The ankle-link antigen: an epitope sensitive to calcium chelation associated with the hair-cell surface and the calycal processes of photoreceptors. J Neurosci 19:3761-3772.

Goodyear RJ, Richardson GP (2003) A novel BAPTA-sensitive antigen as- 
sociated with the tip links and kinocilial links of sensory hair bundles. J Neurosci 23:4878-4887.

Hillman DE (1969) New ultrastructural findings regarding a vestibular ciliary apparatus and its possible functional significance. Brain Res 13:407-412.

Holt JR, Corey DP (2000) Two mechanisms for transducer adaptation in vertebrate hair cells. Proc Natl Acad Sci USA 97:11730-11735.

Howard J, Hudspeth AJ (1987) Mechanical relaxation of the hair bundle mediates adaptation in mechanoelectrical transduction by the bullfrog's saccular hair cell. Proc Natl Acad Sci USA 84:3064-3068.

Jacobs RA, Hudspeth AJ (1990) Ultrastructural correlates of mechanoelectrical transduction in hair cells of the bullfrog's internal ear. Cold Spring Harb Symp Quant Biol 55:547-561.

Kachar B, Parakkal, Kurc M, Zhao Y, Gillespie PG (2000) High resolution structure of hair-cell tip links. Proc Natl Acad Sci USA 97:13336-13341.

Katori Y, Hackney CM, Furness DN (1996) Immunoreactivity of sensory hair bundles of the guinea-pig cochlea to antibodies against elastin and keratan sulphate. Cell Tissue Res 284:473-479.

Kennedy HJ, Evans MG, Crawford AC, Fettiplace R (2003) Fast adaptation of mechanoelectrical transducer channels in mammalian cochlear hair cells. Nat Neurosci 6:832-836.

Killick R, Richardson GP (1997) Antibodies to the sulphated, high molecular mass mouse tectorin stain hair bundles and the olfactory mucus layer. Hear Res 103:131-141.

Kros CJ, Rüsch A, Richardson GP (1992) Mechano-electrical transducer currents in hair cells of the cultured mouse cochlea. Proc R Soc Lond B Biol Sci 249:185-193.

Kros CJ, Lennan GWT, Richardson GP (1995) Transducer currents and bundle movements in outer hair cells of neonatal mice. In: Active hearing (Flock A, Ottoson D, Ulfendahl M, eds), Wenner-Gren International Series, Vol 65, pp 113-125. Oxford: Elsevier.

Kros CJ, Marcotti W, van Netten SM, Self TJ, Libby RT, Brown SD, Richardson GP, Steel KP (2002) Reduced climbing and increased slipping adaptation in cochlear hair cell of mice with Myo7a mutations. Nat Neurosci 5:41-47.

Kruger RP, Goodyear RJ, Legan PK, Warchol ME, Raphael Y, Cotanche DA, Richardson GP (1999) The supporting-cell antigen: a receptor-like protein tyrosine phosphatase expressed in the sensory epithelia of the avian inner ear. J Neurosci 19:4815-4827.

Littlewood Evans A, Muller U (2000) Stereocilia defects in the sensory hair cells of the inner ear in mice deficient in integrin $\alpha 8 \beta 1$. Nat Genet 17:268-269.

Malumbres M, Mangues R, Ferrer N, Lu S, Pellicer A (1997) Isolation of high molecular weight DNA for reliable genotyping of transgenic mice. Biotechniques 22:1114-1119.

Marcotti W, Kros CJ (1999) Developmental expression of the potassium current $I_{\mathrm{KN}}$ contributes to maturation of the mouse outer hair cells. J Physiol (Lond) 520:653-660.

Mitchem KL, Hibbard E, Beyer LA, Bosom K, Dootz GA, Dolan DF, Johnson
KR, Raphael Y, Kohrman DC (2002) Mutation of the novel gene Tmie results in sensory cell defects in the inner ear of spinner, a mouse model of human hearing loss DFNB6. Hum Mol Genet 11:1887-1898.

Nagel G, Neugebauer D-C, Schmidt B, Thurm U (1991) Structures transmitting stimulatory force to the sensory hairs of vestibular ampullae of fishes and frog. Cell Tissue Res 265:567-578.

Neugebauer D-C, Thurm U (1985) Interconnections between the stereovilli of the fish inner ear. Cell Tissue Res 240:449-453.

Norris K, Norris F, Kono DH, Vestergaard H, Pedersen O, Theofilopoulos AN, Møller NPH (1997) Expression of protein-tyrosine phosphatases in the major insulin target tissues. FEBS Lett 415:243-248.

Oganesian A, Poot M, Daum G, Coats SA, Wright MB, Seifert RA, BowenPope DF (2003) Protein tyrosine phosphatase RQ is a phosphatidylinositol phosphatase that can regulate cell survival and proliferation. Proc Natl Acad Sci USA 100:7563-7568.

Pickles JO, Comis SD, Osborne MP (1984) Cross links between stereocilia in the guinea pig organ of Corti and their possible relation to sensory transduction. Hear Res 15:103-112.

Probst FJ, Fridell RA, Raphael Y, Saunders TL, Wang A, Liang Y, Morell RJ, Touchman JW, Lyons RH, Noben-Trauth K, Friedman TB, Camper SA (1998) Correction of deafness in shaker-2 mice by an unconventional myosin in a BAC transgene. Science 280:1444-1447.

Richardson GP, Bartolami S, Russell IJ (1990) Identification of a $275 \mathrm{kDa}$ protein associated with the apical surfaces of sensory hair cells in the avian inner ear. J Cell Biol 110:1055-1066.

Schneider ME, Belyantseva IA, Azevedo RB, Kachar B (2002) Rapid renewal of auditory hair bundles. Nature 418:837-838.

Seifert RA, Coats SA, Oganesian A, Wright MB, Dishmon M, Booth CJ, Johnson RJ, Alpers CE, Bowen-Pope DF (2003) PTPRQ is a novel phosphatidylinositol phosphatase that can be expressed as a cytoplasmic protein or as a subcellularly localized receptor-like protein. Exp Cell Res 287:374-386.

Self T, Mahoney M, Fleming J, Walsh J, Brown SDM, Steel KP (1998) Shaker-1 mutations reveal roles for myosin VIIA in both development and function of cochlear hair cells. Development 125:557-566.

Takenawa T, Itoh T (2001) Phosphoinositides, key molecules for regulation of actin cytoskeletal organisation and membrane traffic from the plasma membrane. Biochem Biophys Acta 1533:190-206.

Wright MB, Hugo C, Seifert R, Disteche CM, Bowen-Pope DF (1998) Proliferating and migrating mesangial cells responding to injury express a novel receptor protein-tyrosine phosphatase in experimental mesangial proliferative glomerulonephritis. J Biol Chem 273:23929-23937.

Wu YC, Ricci AJ, Fettiplace R (1999) Two components of transducer adaptation in auditory hair cells. J Neurophysiol 82:2171-2181.

Zheng L, Sekerkova G, Vranich K, Tilney LG, Mugnaini E, Bartles JR (2000) The deaf jerker mouse has a mutation in the gene encoding the espin actin-bundling proteins of hair cell stereocila and lacks espins. Cell 102: 377-385. 